NASA/CR-1999-208874

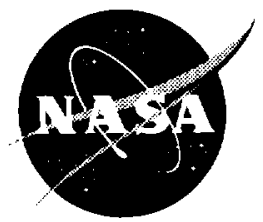

\title{
Analysis of an Interface Crack for a Functionally Graded Strip Sandwiched Between Two Homogeneous Layers of Finite Thickness
}

N.I. Shbeeb and W.K. Binienda

University of Akron, Akron, Ohio

Prepared under Grant NAG3-2201

National Aeronautics and

Space Administration

Lewis Research Center 


\section{Acknowledgments}

This work is sponsored in part by NASA Lewis Research Center Grant NAG3-2201 and the University of Akron. The support from the program manager, Dr. Gary Halford, of NASA Lewis Research Center is appreciated.

Available from

NASA Center for Aerospace Information 7121 Standard Drive Hanover, MD 21076

Price Code: A03
National Technical Information Service 5285 Port Royal Road Springfield, VA 22100 Price Code: A03 


\title{
ANALYSIS OF AN INTERFACE CRACK FOR A FUNCTIONALLY GRADED STRIP SANDWICHED BETWEEN TWO HOMOGENEOUS LAYERS OF FINITE THICKNESS
}

\author{
N.I. Shbeeb and W.K. Binienda \\ University of Akron \\ Department of Civil Engineering \\ Akron, Ohio
}

\begin{abstract}
The interface crack problem for a composite layer that consists of a homogeneous substrate, coating and a non-homogeneous interface was formulated for singular integral equations with Cauchy kernels and integrated using the LobattoChebyshev collocation technique. Mixed-mode Stress Intensity Factors and Strain Energy Release Rates were calculated. The Stress Intensity Factors were compared for accuracy with relevant results previously published. The parametric studies were conducted for the various thickness of each layer and for various non-homogeneity ratios. Particular application to the Zirconia thermal barrier on steel substrate is demonstrated.
\end{abstract}




\section{INTRODUCTION}

Advanced turbine systems and some aerospace applications require use of structural ceramics to protect the hot sections. The thermomechanical mismatch between metal and ceramics induces high residual stresses responsible for cracking and spallation. One way of reduction of the residual stresses is accomplished by processing fully tailored materials and interfacial zones with predetermined, continuously varying mechanical properties known as Functionally Graded Materials (FGM) [1,2]. FGM could be described as twophase particulate composites where the volume fractions of its constituents differ continuously in the thickness direction [3-6].

Erdogan in his paper [7] discussed the problem of crack growth in FGM due to fatigue, creep and stress crack corrosion cracking, and fracture instability.

He concluded the following:

1. By eliminating the discontinuity in material property distributions, the mathematical anomalies regarding the crack tip stress oscillations for the interface cracks are eliminated. Hence, one can now use the crack tip finite element modeling developed for the ordinary square-root singularity and apply the methods of the energy balance-based theories of the conventional fracture mechanics.

2. Application of FGM as interfacial zones in joining generally incompatible materials would greatly improve the bonding strength.

3. Use of FGM as coatings and interfacial zones would reduce the magnitude of the residual and thermal stresses. 
4. Use of FGM coatings and interfaces would eliminate the stress singularities at the points of intersection of interfaces and stress-free ends in bonded materials.

5. Replacing homogeneous coatings by FGM layers would both enhance the bonding strength and reduce the driving forces at the crack tips.

Delale and Erdogan [8] solved the crack problem for a nonhomogeneous plate. The authors considered the plane elasticity problem in which the material is isotropic, has a constant Poisson's ratio (v), and the Young's modulus (E) is of an exponential form varying in the x-direction. They found that the Poisson's ratio did not have much effect on the resulting stress intensity factors. They also found that the strain-energy release rate of the crack embedded in the portion of the medium with higher stiffness is lower than that corresponding to the crack tip in the less stiff side of the material. Hence, the crack will grow in the direction of the less stiff material.

Delale and Erdogan [9] considered the interface crack in a nonhomogeneous elastic medium. In this paper the interface crack between two bonded half planes was addressed. One of the half planes was homogeneous while the other was nonhomogeneous in a manner that the elastic properties are continuous throughout the plane and have discontinuous derivatives along the interface. They assumed that the Young's modulus and the Poisson's ratio are of the exponential form. They found that the singular behavior of the stress state near the crack tip in the nonhomogeneous medium is identical to that in a homogeneous material given that the spatial distribution of the material properties are continuous near and at the crack tip.

Also, Delale and Erdogan [10] solved the crack problem for two bonded dissimilar homogeneous elastic half-planes and assumed that the interfacial region, can 
be modeled by a very thin non-homogeneous layer. The elastic properties of the interfacial material varied continuously between that of the two semi-infinite planes. It was assumed again that $\mathrm{E}$ and $v$ are exponentially dependent on $\mathrm{y}$. Varying the Poisson's ratio did not have much effect on the stress intensity factors. The Airy stress function was used in their formulation of the solution in which it was assumed that it is composed of two functions, one is associated with an infinite plane containing the crack on the $\mathrm{x}$-axis, while the second is an uncracked strip. Their results showed that if the crack location approaches the less stiff material the strain energy release rate increases.

Erdogan et al. [11] studied the perpendicular crack to the interface in a bonded nonhomogeneous material. The main goal was to study how the singular behavior of the stresses and stress intensity factors is affected by the very steep variations in the material properties near the diffusion plane. Here they assumed that the shear modulus $(\mu)$ varies in an exponential form. They suggested that:

1. Regardless of the mechanism of binding at the atomic level, in many cases there is always a thermodynamically stable and readily distinguishable region between the two homogeneous materials.

2. The interfacial regions are generally locations of higher concentration of stress and micro-flaws.

3. In most material pairs the fracture toughness and the sub-critical crack growth resistance of the interfacial zone tend to be lower than that of adjacent homogenous materials.

They found that the nonhomogeneity constant $\beta$ has a great effect on the stress intensity factors. They showed that as $\beta$ increases so do the stress intensity factors. 
Erdogan and Ozturk [12] solved the mixed boundary value problem for a nonhomogeneous medium bonded to a rigid subspace. They investigated a two-dimensional diffusion problem in which the interface contains a plane crack. Rather than solving the problem for a given material, they used an inverse method. This inverse method provided the material constitutive behavior for which the mixed boundary value problem could be solved.

One year later, Erdogan and Ozturk [13] studied the axisymmetric crack problem in a non-homogeneous medium. They noticed that the crack opening displacement in nonhomogeneous materials was significantly greater than the corresponding homogeneous values.

Later Konda and Erdogan [14] considered the mixed mode crack problem in a nonhomogeneous elastic medium. The crack was arbitrarily oriented with respect to the direction of the property gradient. The effect of the variation of the Poisson's ratio was neglected in the solution because of previous studies.

Erdogan and $\mathrm{Wu}[15]$ studied the crack problem in FGM layers under thermal stresses. They considered an unconstrained elastic layer under statically self-equilibrating thermal or residual stresses. The layer contained an embedded or surface crack perpendicular to its boundaries. After giving the distribution of thermal stresses, the stress intensity factors for the embedded and surface crack were presented along with the results of the crack/contact problem in a FGM layer that was under compression near and at the surface and tension in the interior region.

Chen and Erdogan [16] solved the interface crack problem for a nonhomogeneous ceramics coating bonded to a homogenous metallic substrate using displacement 
formulation. It was concluded in this work that the dominant mode of the stress intensity factor is Mode I, and it decreases as the nonhomogeneity constant changes from soft to stiff under uniform normal stress. A similar trend was noticed for Mode II, under uniform shear stress. Decreasing the thickness of either material increased the stress intensity factors.

In this work the problem described in Chen and Erdogan [16] will be extended to include the third thin layer of homogeneous ceramics material to increase thermal protection of the metallic substrate. As shown by Kokini and Choule [17], thermal barriers always include some thickness of pure ceramics material. The scope of this work includes an examination of debonding of ceramics layers from the substrate. In particular, the stress intensity factors (SIF) and strain energy release rates (SERR) are obtained using the Airy stress function formulation for the interface crack embedded between the finite thickness substrate and the non-homogeneous strip, which is sandwiched between the substrate layer and thin homogeneous layer. It is assumed that the FGM has a constant Poisson's ratio and the shear modulus is of an exponential form. Plane elasticity is assumed and the solution is valid for both plane stress and plane strain. 


\section{FORMULATION}

The geometry of the problem is shown in Figure 1. Both the substrate and the coating, which are perfectly bonded to the FGM, are isotropic and homogeneous, and have $h_{1}$ and $h_{3}$ as their respective thickness. The FGM thickness is $h_{2}$, and is denoted as material 2.

In the global $x-y$ coordinates the shear modulus of the FGM is assumed to be as follows:

$$
\mu_{2}(\mathrm{y})=\mu_{1} e^{\gamma \mathrm{y}}
$$

where

$$
\gamma=\frac{1}{h_{2}} \ln \left(\frac{\mu_{3}}{\mu_{1}}\right)
$$

Hooke's law relates strain and stress using two independent material constants:

$$
\begin{aligned}
& \varepsilon_{u x}(x, y)=\frac{\partial u}{\partial x}=\frac{1}{8 \mu}\left[(\kappa+1) \sigma_{x x}+(\kappa-3) \sigma_{y y}\right] \\
& \varepsilon_{y y}(x, y)=\frac{\partial v}{\partial y}=\frac{1}{8 \mu}\left[(\kappa-3) \sigma_{u}+(\kappa+1) \sigma_{y}\right] \\
& \varepsilon_{x y}(x, y)=\frac{1}{2}\left(\frac{\partial u}{\partial y}+\frac{\partial v}{\partial x}\right)=\frac{1}{2 \mu} \tau_{x y}
\end{aligned}
$$

where $\mathrm{u}$ and $\mathrm{v}$ are horizontal and vertical displacements, and $\mathrm{\kappa}$ is defined as:

$$
\begin{array}{ll}
\kappa=3-4 v & \text { for } \text { plane strain } \\
\kappa=\frac{3-v}{1+v} & \text { for } \text { plane stress }
\end{array}
$$


The Airy stress function $F(x, y)$ is defined by

$$
\begin{aligned}
& \sigma_{x y}(x, y)=\frac{\partial^{2} F(x, y)}{\partial y_{1}^{2}} \\
& \sigma_{x y}(x, y)=\frac{\partial^{2} F(x, y)}{\partial x_{1}^{2}} \\
& \tau_{x y}(x, y)=-\frac{\partial^{2} F(x, y)}{\partial x \partial y}
\end{aligned}
$$

By incorporating (4) and the strain-stress relations in the following compatibility equation:

$$
\frac{\partial^{2} \varepsilon_{u}}{\partial x^{2}}+\frac{\partial^{2} \varepsilon_{u}}{\partial y^{2}}-2 \frac{\partial^{2} \varepsilon_{x y}}{\partial x \partial y}=0
$$

we obtain the fourth order governing equation for $F(x, y)$ :

$$
\frac{\partial^{4} F_{1}(x, y)}{\partial x^{4}}+2 \frac{\partial^{4} F_{1}(x, y)}{\partial x^{2} \partial y^{2}}+\frac{\partial^{4} F_{1}(x, y)}{\partial y^{4}}=0
$$

The solution of (6) is found by applying the Fourier Transform:

$$
V(\alpha, y)=\int_{-\infty}^{\infty} F_{1}(x, y) e^{-i \alpha x} d x
$$

that transforms (6) into 4-th order Ordinary Differential Equation (ODE) with constant coefficients. The ODE is solved by forming the characteristic equation to determine its roots:

$$
\begin{aligned}
& \alpha^{4} V-2 \alpha^{2} \frac{d^{2} V}{d y^{2}}+\frac{d^{4} V}{d y^{4}}=0 \\
& \Rightarrow w^{4}-2 \alpha^{2} w^{2}+\alpha^{4}=0 \\
& \Rightarrow w_{1}=w_{3}=|\alpha| \& w_{2}=w_{4}=-|\alpha|
\end{aligned}
$$


Thus, the stress function for the substrate has a form with double repeated roots as follows:

$$
F_{1}(x, y)=\frac{1}{2 \pi} \int_{-\infty}^{\infty}\left[\left(D_{1}(\alpha)+y D_{2}(\alpha)\right) e^{|\alpha| y}+\left(D_{3}(\alpha)+y D_{4}(\alpha)\right) e^{-|\alpha| y}\right] e^{i x \alpha} d \alpha
$$

Similarly, for the coating,

$$
F_{3}(x, y)=\frac{1}{2 \pi} \int_{-\infty}^{\infty}\left[\left(C_{1}(\alpha)+y C_{2}(\alpha)\right) e^{|\alpha| y}+\left(C_{3}(\alpha)+y C_{4}(\alpha)\right) e^{-|\alpha|_{y}}\right] e^{i x \alpha} d \alpha
$$

The governing equation for the FGM is:

$$
\begin{gathered}
\nabla^{2}\left(\sigma_{x}+\sigma_{y}\right)+\gamma^{2}\left[\sigma_{x x}+\frac{\kappa_{2}-3}{\kappa_{2}+1} \sigma_{y}\right]-2 \gamma \frac{\partial}{\partial y}\left(\sigma_{x y}+\sigma_{y y}\right)=0 \\
\Rightarrow \nabla^{4} F_{2}(x, y)+\gamma^{2}\left[\frac{\partial^{2} F_{2}(x, y)}{\partial y^{2}}+\frac{\kappa_{2}-3}{\kappa_{2}+1} \frac{\partial^{2} F_{2}(x, y)}{\partial x^{2}}\right]-2 \gamma \frac{\partial}{\partial y} \nabla^{2} F_{2}(x, y)=0
\end{gathered}
$$

The characteristic equation of (11) becomes,

$$
m^{4}-2 \gamma m^{3}+\left(\gamma^{2}-2 \alpha^{2}\right) m^{2}+2 \gamma \alpha^{2} m+\left(\alpha^{4}-\alpha^{2} \gamma^{2} \frac{\kappa_{2}-3}{\kappa_{2}+1}\right)=0
$$

There are four independent roots of (12):

$$
\begin{aligned}
& m_{1}=\frac{\gamma}{2}-\sqrt{\alpha^{2}+\frac{\gamma^{2}}{4}+i \alpha \gamma \sqrt{\frac{3-\kappa_{2}}{\kappa_{2}+1}}} \\
& m_{2}=\frac{\gamma}{2}+\sqrt{\alpha^{2}+\frac{\gamma^{2}}{4}+i \alpha \gamma \sqrt{\frac{3-\kappa_{2}}{\kappa_{2}+1}}} \\
& m_{3}=\frac{\gamma}{2}-\sqrt{\alpha^{2}+\frac{\gamma^{2}}{4}-i \alpha \gamma \sqrt{\frac{3-\kappa_{2}}{\kappa_{2}+1}}} \\
& m_{4}=\frac{\gamma}{2}+\sqrt{\alpha^{2}+\frac{\gamma^{2}}{4}-i \alpha \gamma \sqrt{\frac{3-\kappa_{2}}{\kappa_{2}+1}}}
\end{aligned}
$$

Consequently, the stress function is obtained in the following form:

$$
F_{2}(x, y)=\frac{1}{2 \pi} \int_{-\infty}^{\infty}\left[A_{1}(\alpha) e^{m_{1} !}+A_{2}(\alpha) e^{m_{2} y}+A_{3}(\alpha) e^{m_{3,} y}+A_{4}(\alpha) e^{m_{1} v}\right] e^{i n \alpha} d \alpha
$$


Using (4) the stresses for the FGM are:

$$
\begin{aligned}
& \sigma_{u}^{(2)}(x, y)=\frac{1}{2 \pi} \int_{-\infty}^{\infty}\left[m_{1}^{2} A_{1} e^{m_{1} y}+m_{2}^{2} A_{2} e^{m_{2} y}+m_{3}^{2} A_{3} e^{m_{2} y}+m_{4}^{2} A_{4} e^{m_{4} y}\right] e^{i x \alpha} d \alpha \\
& \sigma_{y x}^{(2)}(x, y)=-\frac{1}{2 \pi} \int_{-\infty}^{\infty} \alpha^{2}\left[A_{1} e^{m_{1} y}+A_{2} e^{m_{2} y}+A_{3} e^{m_{3} y}+A_{4} e^{m_{4} y}\right] e^{i x \alpha} d \alpha \\
& \tau_{x y}^{(2)}(x, y)=-\frac{1}{2 \pi} \int_{-\infty}^{\infty} i \alpha\left[m_{1} A_{1} e^{m_{1} y}+m_{2} A_{2} e^{m_{2} y}+m_{3} A_{3} e^{m_{3 y} y}+m_{4} A_{4} e^{m_{4} y}\right] e^{i x \alpha} d \alpha
\end{aligned}
$$

the stresses for the substrate are:

$$
\begin{aligned}
& \sigma_{u x}^{(\prime)}(x, y)=\frac{1}{2 \pi} \int_{-\infty}^{\infty}\left[\left(\alpha^{2}\left(D_{1}+y D_{2}\right)+2|\alpha| D_{2}\right) e^{|\alpha| y}+\left(\alpha^{2}\left(D_{3}+y D_{4}\right)-2|\alpha| D_{4}\right) e^{-|\alpha| y}\right] e^{i x \alpha} d \alpha \\
& \left.\left.\sigma_{y y}^{(\prime \prime}(x, y)=-\frac{1}{2 \pi} \int_{-\infty}^{\infty} \alpha^{2}\left[\left(D_{1}+y D_{2}\right)\right] e^{|\alpha| y}+\left(D_{3}+y D_{4}\right)\right] e^{-|\alpha| y}\right] e^{i x \alpha} d \alpha \\
& \tau_{u y}^{(1)}(x, y)=-\frac{1}{2 \pi} \int_{-\infty}^{\infty} i \alpha\left[\left(|\alpha|\left(D_{1}+y D_{2}\right)+D_{2}\right) e^{|\alpha| y}+\left(-|\alpha|\left(D_{3}+y D_{4}\right)+D_{4}\right) e^{-|\alpha| y}\right] e^{i x \alpha} d \alpha
\end{aligned}
$$

and for the coating they are as follows:

$$
\begin{aligned}
& \sigma_{u}^{(3)}(x, y)=\frac{1}{2 \pi} \int_{-\infty}^{\infty}\left[\left(\alpha^{2}\left(C_{1}+y C_{2}\right)+2|\alpha| C_{2}\right) e^{|\alpha| y}+\left(\alpha^{2}\left(C_{3}+y C_{4}\right)-2|\alpha| C_{4}\right) e^{-|\alpha| y}\right] e^{i x \alpha} d \alpha \\
& \left.\sigma_{n}^{(3)}(x, y)=-\frac{1}{2 \pi} \int_{-\infty}^{\infty} \alpha^{2}\left[\left(C_{1}+y C_{2}\right)\right] e^{|\alpha| y}+\left(C_{3}+y C_{4}\right) e^{-|\alpha| y}\right] e^{i x \alpha} d \alpha \\
& \tau_{n y}^{(3)}(x, y)=-\frac{1}{2 \pi} \int_{-\infty}^{\infty} i \alpha\left[\left(|\alpha|\left(C_{1}+y C_{2}\right)+C_{2}\right) e^{|\alpha| y}+\left(-|\alpha|\left(C_{3}+y C_{4}\right)+C_{4}\right) e^{-|\alpha| y}\right] e^{i x \alpha} d \alpha
\end{aligned}
$$

From (15), (16) and (17), it can be seen that there are 12 unknown constants (in the Fourier space they are functions of $\alpha$ ), i.e., $D_{i}, C_{i}$ and $A_{i}(i=1 \ldots 4)$, which can be obtained from the following conditions:

$$
\begin{aligned}
& \sigma_{w}^{(1)}\left(x,-h_{1}\right)=0, \tau_{v y}^{(1)}\left(x,-h_{1}\right)=0 \\
& \sigma_{w}^{(2)}(x, 0)=\sigma_{w}^{(1)}(x, 0), \tau_{x y}^{(2)}(x, 0)=\tau_{x y}^{(1)}(x, 0) \\
& \sigma_{w}^{(3)}\left(x, h_{2}+h_{3}\right)=0, \tau_{v y}^{(3)}\left(x, h_{2}+h_{3}\right)=0 \\
& \sigma_{w}^{(2)}\left(x, h_{2}\right)=\sigma_{v}^{(3)}\left(x, h_{2}\right), \tau_{n}^{(2)}\left(x, h_{2}\right)=\tau_{v}^{(3)}\left(x, h_{2}\right) \\
& u^{(2)}\left(x, h_{2}\right)=u^{(3)}\left(x, h_{2}\right), v^{(2)}\left(x, h_{2}\right)=v^{(3)}\left(x, h_{2}\right) \\
& -\infty<x<\infty
\end{aligned}
$$


and the following mixed boundary conditions:

$$
\begin{gathered}
\sigma_{y y}^{(2)}\left(x, 0^{+}\right)=p_{1}(x), \tau_{x y}^{(2)}\left(x, 0^{+}\right)=p_{2}(x), \quad-a<x<a \\
u^{(2)}\left(x, 0^{+}\right)-u^{(1)}\left(x, 0^{-}\right)=0, \quad v^{(2)}\left(x, 0^{+}\right)-v^{(1)}\left(x, 0^{-}\right)=0 \quad|x|>a
\end{gathered}
$$

where $p_{1}$ and $p_{2}$ are known surface tractions. In order to convert the solution from a dual integral path into a singular integral equations, $(20 \mathrm{a}, \mathrm{b})$ has to be replaced by the following auxiliary functions:

$$
\begin{aligned}
& \frac{\partial}{\partial x}\left[u^{(2)}\left(x, 0^{+}\right)-u^{(1)}\left(x, 0^{-}\right)\right]=f_{1}(x) \cdots \cdots-\infty<x<\infty \\
& \frac{\partial}{\partial x}\left[v^{(2)}\left(x, 0^{+}\right)-v^{(1)}\left(x, 0^{-}\right)\right]=f_{2}(x) \cdots \cdots-\infty<x<\infty
\end{aligned}
$$

Consequently, all twelve unknowns are determined in terms of the auxiliary functions, $f_{1}(x)$ and $f_{2}(x)$, by using (18) and (21). The unknown auxiliary functions are solved by using (19). After some lengthy manipulations the following singular integral equation (SIE) was reached. For details see [18],

$$
\begin{aligned}
& \frac{\pi\left(1+\kappa_{1}\right)\left(1+\kappa_{2}\right)}{\mu_{1}\left(2+\kappa_{1}+\kappa_{2}\right)}\left(-p_{1}(x)\right)=\int_{-a}^{a} \frac{f_{2}(t)}{t-x} d t+\int_{-a}^{a} f_{1}(t) K_{11}(x, t)+\int_{-a}^{a} f_{2}(t) K_{12}(x, t) \\
& +\frac{\pi \gamma\left(5+5 \kappa_{2}+\kappa_{2}^{2}+\kappa_{1}^{2}\left(2+\kappa_{2}\right)+\kappa_{1}\left(6+4 \kappa_{2}\right)\right)}{4\left(1+\kappa_{1}\right)\left(1+\kappa_{2}\right)\left(2+\kappa_{1}+\kappa_{2}\right)} \int_{-a}^{a} \frac{(t-x)}{|t-x|} f_{2}(t) d t \\
& -\frac{\gamma\left(1-\kappa_{2}-\kappa_{2}^{2}+2 \kappa_{1}\left(2+\kappa_{2}\right)+\kappa_{1}^{2}\left(2+\kappa_{2}\right)\right)}{2\left(1+\kappa_{1}\right)\left(1+\kappa_{2}\right)\left(2+\kappa_{1}+\kappa_{2}\right)} \int_{-a}^{a} C i(U(t-x)) f_{1}(t) d t \\
& \frac{\pi\left(1+\kappa_{1}\right)\left(1+\kappa_{2}\right)}{\mu_{1}\left(2+\kappa_{1}+\kappa_{2}\right)}\left(-p_{2}(x)\right)=\int_{-a}^{a} \frac{f_{1}(t)}{t-x} d t+\int_{-a}^{a} f_{1}(t) K_{21}(x, t)+\int_{-a}^{a} f_{2}(t) K_{22}(x, t) \\
& +\frac{\pi \gamma\left(1+2 \kappa_{2}+\kappa_{2}+\kappa_{2}^{2}\left(2+\kappa_{2}\right)+\kappa_{1}^{2}\left(2+\kappa_{2}\right)\right)}{2\left(1+\kappa_{1}\right)\left(1+\kappa_{2}\right)\left(2+\kappa_{1}+\kappa_{2}\right)} \int_{-a}^{a} \frac{(t-x)}{|t-x|} f_{1}(t) d t \\
& +\frac{\gamma\left(1-\kappa_{2}-\kappa_{2}^{2}+2 \kappa_{1}\left(2+\kappa_{2}\right)+\kappa_{1}^{2}\left(2+\kappa_{2}\right)\right)}{2\left(1+\kappa_{1}\right)\left(1+\kappa_{2}\right)\left(2+\kappa_{1}+\kappa_{2}\right)} C i(U(t-x)) f_{2}(t) d t
\end{aligned}
$$


where,

$$
\begin{aligned}
& K_{11}(x, t)=\frac{\left(1+\kappa_{1}\right)\left(1+\kappa_{2}\right)}{2\left(2+\kappa_{1}+\kappa_{2}\right)}\left\{\int_{0}^{\infty}\left(x_{11 \mathrm{c}}-x_{11}\right) i \sin (\alpha(t-x)) d \alpha+\right. \\
& \int_{0}^{U}\left(x_{11}+x_{1 \mathrm{t}}-2\left(\frac{\kappa_{1}-\kappa_{2}}{\left(1+\kappa_{1}\right)\left(1+\kappa_{2}\right)}\right)\right) \cos (\alpha(t-x)) d \alpha+\int_{U}^{\infty}\left(x_{11}+x_{1 \mathrm{t}}\right. \\
& \left.\left.-2\left(\frac{\kappa_{1}-\kappa_{2}}{\left(1+\kappa_{1}\right)\left(1+\kappa_{2}\right)}+\frac{\gamma\left(1+4 \kappa_{1}+2 \kappa_{1}^{2}-\kappa_{2}+2 \kappa_{1} \kappa_{2}+\kappa_{1}^{2} \kappa_{2}-\kappa_{2}^{2}\right.}{2 \alpha\left(1+\kappa_{1}\right)^{2}\left(1+\kappa_{2}\right)^{2}}\right)\right) \cos (\alpha(t-x)) d \alpha\right\} \\
& \mathrm{K}_{12}(\mathrm{x}, \mathrm{t})=\frac{\left(1+\kappa_{1}\right)\left(1+\kappa_{2}\right)}{2\left(2+\kappa_{1}+\kappa_{2}\right)}\left\{\int _ { 0 } ^ { \infty } \left(\mathrm{x}_{12 \mathrm{c}}-\mathrm{x}_{12}+2 \mathrm{i}\left(\frac{2+\kappa_{1}+\kappa_{2}}{\left(1+\kappa_{1}\right)\left(1+\kappa_{2}\right)}+\right.\right.\right. \\
& \left.\frac{\gamma\left(5+6 \kappa_{1}+2 \kappa_{1}^{2}+5 \kappa_{2}+4 \kappa_{1} \kappa_{2}+\kappa_{1}^{2} \kappa_{2}+\kappa_{2}^{2}\right.}{2 \alpha\left(1+\kappa_{1}\right)^{2}\left(1+\kappa_{2}\right)^{2}}\right) i \sin (\alpha(t-x)) d \alpha \\
& \left.+\int_{0}^{\infty}\left(x_{12}+x_{12 c}\right) \cos (\alpha(t-x)) d \alpha\right\} \\
& \mathrm{K}_{21}(\mathrm{x}, \mathrm{t})=\frac{\left(1+\kappa_{1}\right)\left(1+\kappa_{2}\right)}{2\left(2+\kappa_{1}+\kappa_{2}\right)}\left\{\int _ { 0 } ^ { \infty } \left(\mathrm{x}_{21 \mathrm{i}}-\mathrm{x}_{21}+2 \mathrm{i}\left(\frac{2+\kappa_{1}+\kappa_{2}}{\left(1+\kappa_{1}\right)\left(1+\kappa_{2}\right)}+\right.\right.\right. \\
& \left.\frac{\gamma\left(1+2 \kappa_{1}+2 \kappa_{1}^{2}+\kappa_{2}+\kappa_{1}^{2} \kappa_{2}+\kappa_{2}^{2}\right.}{2 \alpha\left(1+\kappa_{1}\right)^{2}\left(1+\kappa_{2}\right)^{2}}\right) i \sin (\alpha(t-x)) d \alpha \\
& \left.+\int_{0}^{\infty}\left(x_{21}+x_{21 c}\right) \cos (\alpha(t-x)) d \alpha\right\} \\
& K_{22}(x, t)=\frac{\left(1+\kappa_{1}\right)\left(1+\kappa_{2}\right)}{2\left(2+\kappa_{1}+\kappa_{2}\right)}\left\{\int_{0}^{\infty}\left(x_{22 c}-x_{22}\right) i \sin (\alpha(t-x)) d \alpha+\right. \\
& \int_{0}^{u}\left(x_{22}+x_{22 c}-2\left(\frac{\kappa_{1}-\kappa_{2}}{\left(1+\kappa_{1}\right)\left(1+\kappa_{2}\right)}\right)\right) \cos (\alpha(t-x)) d \alpha+\int_{u}^{\infty}\left(x_{11}+x_{11 c}\right. \\
& \left.\left.-2\left(-\frac{\kappa_{1}-\kappa_{2}}{\left(1+\kappa_{1}\right)\left(1+\kappa_{2}\right)}-\frac{\gamma\left(1+4 \kappa_{1}+2 \kappa_{1}^{2}-\kappa_{2}+2 \kappa_{1} \kappa_{2}+\kappa_{1}^{2} \kappa_{2}-\kappa_{2}^{2}\right.}{2 \alpha\left(1+\kappa_{1}\right)^{2}\left(1+\kappa_{2}\right)^{2}}\right)\right) \cos (\alpha(t-x)) d \alpha\right\}
\end{aligned}
$$




$$
\begin{aligned}
& x_{11}=-\alpha^{2}\left(\frac{Q_{11}}{Q}-\frac{Q_{21}}{Q}+\frac{Q_{31}}{Q}-\frac{Q_{41}}{Q}\right) \\
& x_{12}=-\alpha^{2}\left(-\frac{Q_{12}}{Q}+\frac{Q_{22}}{Q}-\frac{Q_{32}}{Q}+\frac{Q_{42}}{Q}\right) \\
& x_{21}=-i \alpha\left(m_{1} \frac{Q_{11}}{Q}-m_{2} \frac{Q_{21}}{Q}+m_{3} \frac{Q_{31}}{Q}-m_{4} \frac{Q_{41}}{Q}\right) \\
& x_{22}=-i \alpha\left(-m_{1} \frac{Q_{12}}{Q}+m_{2} \frac{Q_{22}}{Q}-m_{3} \frac{Q_{32}}{Q}+m_{4} \frac{Q_{42}}{Q}\right) \\
& x_{11 c}=\operatorname{conjugate}\left(x_{11}\right), x_{12 c}=\operatorname{conjugate}\left(x_{12}\right) \\
& x_{21 c}=\operatorname{conjugate}\left(x_{21}\right), x_{22 c}=\operatorname{conjugate}\left(x_{22}\right) \\
& \mathrm{Ci}\left(\mathrm{U}\left(\mathrm{t}-\mathrm{x}_{1}\right)\right)=\left(\mathrm{C}_{0}+\log \left|\mathrm{U}\left(\mathrm{t}-\mathrm{x}_{1}\right)\right|+\int_{0}^{\mid \mathrm{U}\left(t-x_{1}||\right.} \frac{\cos \beta-1}{\beta} \mathrm{d} \beta\right)
\end{aligned}
$$

$\mathrm{C}_{0}$ is the Euler constant, whereas $\mathrm{U}$ is the upper limit beyond which the cosine integral, $\mathrm{Ci}$, is negligible. $\mathrm{Q}$ is the determinant of the 4 by 4 coefficient matrix and $\mathrm{Q}_{\mathrm{ij}}$ are the corresponding 3 by 3 cofactors. Both can be found in the Appendix. 


\section{SINGULAR INTEGRAL EQUATIONS SOLUTION}

The singular integral equations (22) contain Cauchy kernels. In order to obtain unique results the following conditions need to be incorporated into the solution.

$$
\int_{-a}^{a} f_{i}(t) d t=0 \cdots \cdots \cdot i=1,2
$$

The system of equations (22) and (29) can be solved together using the Gauss quadrature method. For example, using, the Lobatto-Chebyshev collocation method was shown in [19] to produce accurate results for the above type of equations. In practice the auxiliary functions are discretized at particular points $\mathrm{t}_{\mathrm{k}}$, integration is replaced by a summation, and the system of linear algebraic equations are obtained for collocation points $x_{p}\left(x_{p}\right.$ is never the same as $\left.t_{k}\right)$ in the following form:

$$
\sum_{j=1}^{N} \frac{b_{i j}}{\pi} \sum_{k=1}^{n} \frac{g_{j}\left(t_{k}\right) w_{k}}{t_{k}-x_{p}}+\sum_{j=1}^{N} \sum_{k=1}^{n} k_{i j}\left(x_{p}, t_{k}\right) g_{j}\left(t_{k}\right) w_{k}+R_{n}\left(x_{p}\right)=f_{i}\left(x_{p}\right)
$$

where $p=1, \ldots, n, w_{k}$ is the weighting coefficient, and $R_{n}$ is a remainder that becomes small for sufficiently large number of points $t_{k}$. According to the aforementioned technique, the abscissas are calculated according to:

$$
t_{k}=\cos \left(\frac{(k-1) \pi}{n-1}\right) \cdots \cdots k=1, \ldots, n .
$$

The corresponding weights are:

$$
w_{1}=w_{n}=\frac{\pi}{2(n-1)} ; w_{r}=\frac{\pi}{n-1} \cdots \cdots r=2, \ldots, n-1 .
$$

The collocation points can be found from:

$$
x_{p}=\cos \left(\frac{(2 p-1) \pi}{2 n-2}\right) \cdots \cdots p=1, \ldots, n-1 .
$$


The remaining two equations are generated using (29) in the following form:

$$
\begin{aligned}
& \sum_{k=1}^{n} g_{1}\left(s_{k}\right) w_{k}=0 \\
& \sum_{k=1}^{n} g_{2}\left(s_{k}\right) w_{k}=0
\end{aligned}
$$

By incorporating (30), (34) and (33) together, the system of equations can be represented as follows:

$$
[A]_{2 n \times 2 n}\{g\}_{2 n}=\{P\}_{2 n}
$$

thus, the unknowns are obtained by:

$$
\{g\}=[A]^{-1}\{P\}
$$

The mode-I and mode-II SIF are defined as follows:

$$
\begin{aligned}
& k_{1}(a)=\lim _{x_{1} \rightarrow a} \sqrt{2\left(x_{1}-a\right)} \sigma_{y_{1} y_{1}}\left(x_{1}, 0\right) \\
& k_{2}(a)=\lim _{x_{1} \rightarrow a} \sqrt{2\left(x_{1}-a\right)} \tau_{x_{1} y_{1}}\left(x_{1}, 0\right)
\end{aligned}
$$

From the principal part of the expressions for $g_{1}(t)$ and $g_{2}(t)$ as shown in [20], the following are obtained for $k_{1}(a)$ and $k_{2}(a)$ :

$$
\begin{gathered}
k_{1}(a)=\frac{\mu_{1}\left(2+\kappa_{1}+\kappa_{2}\right)}{\left(1+\kappa_{1}\right)\left(1+\kappa_{2}\right) \sqrt{a}} g_{2}(a) \\
k_{2}(a)=-\frac{\mu_{1}\left(2+\kappa_{1}+\kappa_{2}\right)}{\left(1+\kappa_{1}\right)\left(1+\kappa_{2}\right) \sqrt{a}} g_{1}(a)
\end{gathered}
$$

Where $g_{1}(a)$ and $g_{2}(a)$ correspond to $g_{1}(1)$ and $g_{2}(1)$ respectively when solving (36).

The strain energy release rates (SERR) can be calculated from [16], and they are listed as follows:

$$
\begin{aligned}
& G_{1}(a)=\frac{\pi\left(\kappa_{2}+1\right)}{8 \mu_{1}} k_{1}(a)^{2} \\
& G_{2}(a)=\frac{\pi\left(\kappa_{2}+1\right)}{8 \mu_{1}} k_{2}(a)^{2}
\end{aligned}
$$


where $G_{1}$ and $G_{2}$ are the opening mode and sliding mode SERR, respectively. The total SERR is expressed as:

$$
G_{T}(a)=\frac{\pi\left(\kappa_{2}+1\right)}{8 \mu_{1}}\left(k_{1}(a)^{2}+k_{2}(a)^{2}\right)
$$

Before performing the parametric study the above solution should be verified with an existing solution close to the proposed problem. The verification is accomplished by comparing the results of our model with that of [16] by letting $h_{3}$ approach zero and $\gamma a$ (the normalized non-homogeneity constant with respect to the crack length) taking the values as shown in the Table I.

The values were obtained for $h_{1}=2 a, h_{2}=a, v_{1}=v_{2}=v_{3}=0.3$ under loading of uniform normal stress. The results in Table 1 demonstrate the accuracy of the solution.

\section{RESULTS}

The geometry of the problem being examined is shown in Figure 1. The thickness of each layer is normalized with respect to the half-length of the crack "a" located at the interface between the substrate layer and FGM. The homogeneous substrate, material "1", may be stiffer or softer with respect to the homogeneous layer of ceramics, material "3". The normalized nonhomogeneity constant $\gamma \mathrm{a}$ is varied between -3 and 3 , which covers most of the practical cases. The results are calculated for normalized mode-I and mode-II SIFs, i.e., $k_{1} / k_{0}$ and $k_{2} / k_{0}$, and normalized SERR, i.e., $G_{1} / G_{0}$ and $G_{2} / G_{0}$, where $k_{0}=\sigma_{n} \sqrt{c}$ and $G_{0}=\frac{8 \mu_{0} k_{0}^{2}}{\pi\left(\kappa_{2}+1\right)}$. The results are calculated for two loading conditions, namely unit normal stress in y direction and unit shear stress in $x-y$ plane. The Poisson's ratio is assumed to be $v=0.3$ for each layer. 
First, the influence of the additional ceramics layer will be examined in order to determine if there is any advantage to fabricate a thermal barrier with pure ceramics on top of the FGM layer. Figures 2 and 3 show normalized total SERR versus the thickness of the homogeneous ceramic layer $h_{3}$ produced by normal stress and shear stress, respectively. The thickness of the substrate is assumed 4 times higher than the thickness of the FGM. The crack length is assumed to be the same as the thickness of FGM layer.

It can be noticed that SERR is significantly reduced even by a small additional thickness of the ceramics material. The rate of reduction is the highest for $h_{3}$ below $0.5 a$ for $h_{3}$ larger than $3 a$, the influence is negligible. The largest reduction is produced for the negative nonhomogeneity constant when the stiffness of the ceramics material is smaller than the substrate. The smallest reduction is in the case of the stiffer ceramic material. The homogeneous case is obtained for $\gamma \mathrm{a}=0$ and its SERR curve is located in between the other two cases shown. One can conclude that the small change of the thickness of the layer above the crack can significantly reduce the SERR. It can be recommended that the optimum thickness of the ceramics layer should be about half of the thickness of the FGM layer.

Using the above recommendation we can compare the SERR with the results obtained by Chen and Erdogan (1996) indicated in the following figures by $h_{3}=0$ (this case is also the limiting case of our model). Figures 4,5 and 6 are generated for the case of the normal applied stress for three different thickness of the FGM layer. The thickness of the substrate is assumed to be very large. Comparison of the curves with the additional layer of ceramics and without that layer $\left(h_{3}=0\right)$ shows that the ceramics layer reduces the SERR for each case, but the most significant reduction is for the thinnest FGM layer. In 
all cases, the magnitude of $G, k_{1}$ and $k_{2}$ decreases as the stiffness of the ceramics layer increases.

Figures 7-9 show SERR and SIF for the case of the applied shear stress for the same geometrical and material conditions as in the Figures 4-6. Similarly as for normal applied stress, $\mathrm{G}, \mathrm{k}_{1}$ and $\mathrm{k}_{2}$ decrease with the increasing nonhomogeneity constant. For the case of the softest ceramic layer, additional thickness $h_{3}=0.125 \mathrm{a}$ of the homogeneous layer added to $0.25 \mathrm{a}$ thick FGM reduces $\mathrm{G}, \mathrm{k}_{1}$ and $\mathrm{k}_{2}$ as much as increasing the thickness from $0.25 \mathrm{a}$ to $0.5 \mathrm{a}$ of the FGM. Hence, either increasing the FGM thickness or increasing the ceramic layer can reduce the SIF and SERR.

Figures 10 and 11 show the normalized total SERR generated by normal and shear stresses, respectively, versus the nonhomogeneity constant for the various thickness of the substrate. The reduction of the SERR by increasing thickness of the ceramic layer is equally significant to each thickness of the substrate. The cases for $h_{1}=10 a$ and $h_{1}=4 a$ overlap for the applied normal stress and they are almost identical for the applied shear stress. As in the previous cases, the stiffness ratio of the ceramics coating to substrate significantly changes the SERR, especially for the thin layer of the substrate.

Mode-I is dominant under normal stress tractions. A question arises what is better under such loading conditions: thicker FGM layer without any homogeneous layer of ceramics or thinner FGM with $\mathrm{h}_{3}$ making the difference. In the last parametric study we will assume that the total thickness of the FGM and homogeneous ceramic coating is constant. The results of SERR are plotted with respect to $\ln \left(\mu_{3} / \mu_{1}\right)$ in Figure 12 .

It can be noticed that for increasing $\ln \left(\mu_{3} / \mu_{1}\right)$ the SERR decreases. The highest $\mathrm{G}$ is for the most negative $\ln \left(\mu_{3} / \mu_{1}\right)$. By replacing part of FGM by pure ceramic material we 
increase $\mathrm{G}$ for $\ln \left(\mu_{3} / \mu_{1}\right)=-2$ (the region above the crack is softened by the softer ceramics layer) and decrease $\mathrm{G}$ slightly for $\ln \left(\mu_{3} / \mu_{1}\right)=2$ (the region above the crack is stiffened by the stiffer ceramics layer).

Hence, it can be concluded that SERR can be reduced by several methods: stiffer coating application, thicker FGM layer, and additional layer of homogeneous ceramics. The most optimum combination depends on the stiffness ratio of the ceramics with respect to the substrate.

Let's apply the above knowledge to the specific cases shown in Figure 13 (also discussed in [17]). The substrate material is steel. Zirconia is used for the ceramic coating. The FGM is made by gradual change from $100 \%$ of Zirconia to Zirconia/CoCrAlY to the bond coat attached to the substrate. In case (a), there are 50/50 of Zirconia/CoCrAlY layer and bond layer sandwiched between pure ceramics and substrate material. In case (b), there are four layers of Zirconia/CoCrAlY, i.e., 75/25, $50 / 50,25 / 75$, and bond layer sandwiched between pure ceramics and substrate material.

The properties of materials are taken from [21]. For Zirconia they are: $E_{3}=36$ GPa and $v_{3}=0.2$, while for steel they are: $E_{1}=207 \mathrm{GPa}$ and $v_{1}=0.33$. The thickness of each layer can be normalized with respect to crack length, which is assumed to be the same as half of the total thermal barrier thickness, i.e., $h_{t}=h_{2}+h_{3}=a$. The thickness of the substrate is $h_{1}=6 a$.

Table II shows results for three different cases under normal stress conditions. In the first case the ceramic layer is part of the FGM. In the second and third cases the pure ceramic layer belongs to material "3" and FGM thickness is measured from the interfaces between steel and bond coat to the interface between Zirconia and FGM layer. It can be 
noticed that the total SERR is higher in the lower two cases than it is in the first case by $16 \%$ and $38 \%$, respectively. Hence, it is critical how FGM is defined in the practical case studies. It can be also noticed that the thinner Zirconia layer produced smaller SERR because of the negative nonhomogeneity ratio as described in Figure 12. Finally, the results show some sensitivity to the FGM Poisson's ratio, but for all practical purposes it can be assumed to be the same as for the pure ceramics.

\section{REFERENCES}

1. Asish Ghosh, Yoshinari Miyamoto, Ivar Reimanis and John J. Lannutti (eds.) Functionally Graded Materials. 1997, Ceramic Trans., 76.

2. Holt J. Birch, Mitsue Koizomi, Toshio Hirai and Zuhir A. Munir (eds.) Functionally Graded Materials. Ceramic Trans., 1993, 34, The American Ceramic Society, Westerville, Ohio.

3. Niino, M. and Maeda, S. Recent Development Status of Functionally Gradient Materials. I. S. I. J. Int. 1990, 30, 699-703.

4. Hirano, T., Yamada, T., Teraki, J., Niino, M. and Kumakawa, A. A Study on Functionally Gradient Material Design System for a Thrust Chamber. Proc. $16^{\text {th }}$ Int. Symp. On Space Technology and Science, 1988, Sapporo, Japan.

5. Hirano, T. and Yamada, T. Multi-Paradigm Expert System Architecture Based Upon the Inverse Design Concept. Int. Workshop on Artificial Intelligence for Industrial Applications, 1988, Hitachi, Japan. 
6. Kawasaki, A. and Watanabe, R. Fabrication of Sintered Functionally Gradient Material by Powder Spray Forming Process. FGM-90, Proc. $1^{\text {st }}$ Int. Symp. on FGM, 1990, Sendai, Japan.

7. Erdogan, F. Fracture Mechanics of Functionally Graded Materials. Composites Engineering, 1995, 5, 753-770.

8. Delale, F., Erdogan, F. The Crack Problem for a Non-Homogeneous Plane. Journal of Applied Mechanics, 1983, 50, 609-614.

9. Delale, F., Erdogan, F. Interface Crack in a Non-Homogeneous Elastic Medium. Int. J., Engng. Sci., 1988, 26, 559-568.

10. Delale, F. and Erdogan, F. On the Mechanical Modeling of the Interfacial Region in Bonded Half-Planes. Journal of Applied Mechanics, 1988, 55, 317-324.

11. Erdogan, F., Kaya, A. C. and Joseph, P. F. The Crack Problem in Bonded NonHomogeneous Materials. Journal of Applied Mechanics, 1991, 58, 410-418.

12. Erdogan, F. and Ozturk, M. Diffusion Problems in Bonded Nonhomogeneous Materials with an interface Cut. Int. J. Engng. Sci., 1992, 30, 1507-1523.

13. Erdogan, F. and Ozturk, M. The Axis-symmetric Crack Problem in a Nonhomogeneous Medium. Journal of Applied Mech., 1993, 60, 406-413.

14. Konda, N. and Erdogan, F. The Mixed Mode Crack Problem in a Non-Homogeneous Elastic Medium. Engineering Fracture Mechanics, 1994, 47, 533-545.

15. Erdogan, F. and Wu, H. B. Crack Problems in FGM Layers under Thermal Stresses. Journal of Thermal Stresses, 1996, 19, 237-265. 
16. Chen, Y. F., Erdogan, F. The Interface Crack Problem for a Non-Homogeneous Coating Bonded to a Homogeneous Substrate. J. Mech. Phys. Solids, 1996, 44, 771787.

17. Kokoni, K. and Choules, B. D. Surface Thermal Fracture of Functionally Graded Ceramic Coatings: Effect of Architecture and Materials, Composites Engineering, $1995,7,865-877$.

18. Shbeeb N. General Crack Problems in Functionally Graded Materials. Ph.D. dissertation, 1998, The University of Akron.

19. Theocraris, P. S., and Ioakimidis, N. I. Numerical Integration Methods for the Solution of Singular Integral Equations. Quarterly of Appl. Math, 1977, 173-183.

20. Muskhelishvili, N. I. Singular Integral Equations. 1953, Nooedhoff, Groningen, The Netherlands.

21. Pindera, M. J., Aboudi, J. and Arnold, S. M. The Effect of Microstructure on the Response of Functionally Graded Thermal Barrier Coatings. Proc. Of the HITEMP Review, 1997, Vol. II: Advanced Alloys and MMC. 
Table I

Verification of the model

\begin{tabular}{|c|c|c|c|c|}
\hline$\gamma \mathrm{a}$ & $\begin{array}{c}\mathrm{k}_{1}(\mathrm{a}) /\left(\sigma_{0} \sqrt{\mathrm{a}}\right) \\
\text { Chen and } \\
\text { Erdogan } \\
(1996)\end{array}$ & $\begin{array}{c}\mathrm{k}_{1}(\mathrm{a}) /\left(\sigma_{0} \sqrt{\mathrm{a}}\right) \\
\text { Present } \\
\text { study }\end{array}$ & $\begin{array}{c}\mathrm{k}_{2}(\mathrm{a}) /\left(\sigma_{0} \sqrt{\mathrm{a}}\right) \\
\text { Chen and } \\
\text { Erdogan } \\
(1996)\end{array}$ & $\begin{array}{c}\mathrm{k}_{2}(\mathrm{a}) /\left(\sigma_{0} \sqrt{\mathrm{a}}\right) \\
\text { Present } \\
\text { study }\end{array}$ \\
\hline-3.0 & 2.430 & 2.428 & -0.681 & -0.624 \\
\hline-2.5 & 2.252 & 2.251 & -0.571 & -0.533 \\
\hline-2.0 & 2.087 & 2.087 & -0.471 & -0.445 \\
\hline-1.5 & 1.936 & 1.936 & -0.379 & -0.364 \\
\hline-1.25 & 1.866 & 1.866 & -0.336 & -0.325 \\
\hline-1.0 & 1.799 & 1.799 & -0.296 & -0.288 \\
\hline-0.75 & 1.735 & 1.735 & -0.258 & -0.252 \\
\hline-0.5 & 1.675 & 1.675 & -0.221 & -0.218 \\
\hline-0.25 & 1.618 & 1.618 & -0.187 & -0.186 \\
\hline-0.01 & 1.566 & 1.566 & -0.156 & -0.155 \\
\hline 0.25 & 1.514 & 1.514 & -0.125 & -0.126 \\
\hline 0.5 & 1.466 & 1.466 & -0.096 & -0.099 \\
\hline 0.75 & 1.422 & 1.422 & -0.069 & -0.072 \\
\hline 1.0 & 1.380 & 1.379 & -0.044 & -0.048 \\
\hline 1.5 & 1.304 & 1.303 & 0.002 & 0.003 \\
\hline 2.0 & 1.237 & 1.237 & 0.042 & 0.036 \\
\hline 2.5 & 1.179 & 1.178 & 0.077 & 0.070 \\
\hline 3.0 & 1.128 & 1.127 & 0.108 & 0.100 \\
\hline
\end{tabular}


Table II

SIF and SERR for Zirconia thermal barrier and steel substrate.

\begin{tabular}{|l|l|l|l|l|l|l|}
\hline $\begin{array}{l}\text { Geometry and } \\
\text { Material } \\
\text { Property }\end{array}$ & \multicolumn{2}{|c|}{$\mathrm{k}_{1}(\mathrm{a})$} & \multicolumn{2}{c|}{$\mathrm{k}_{2}(\mathrm{a})$} & \multicolumn{2}{c|}{$\mathrm{G}_{\mathrm{T}}(\mathrm{a})$} \\
\cline { 2 - 7 } & $v_{2}=\mathrm{v}_{3}$ & $\mathrm{v}_{2}=\mathrm{v}_{1}$ & $\mathrm{v}_{2}=\mathrm{v}_{3}$ & $\mathrm{v}_{2}=\mathrm{v}_{1}$ & $\mathrm{v}_{2}=\mathrm{v}_{3}$ & $\mathrm{v}_{2}=\mathrm{v}_{1}$ \\
\hline $\begin{array}{l}\mathrm{h}_{1}=\mathrm{a}, \mathrm{h}_{2}=\mathrm{a}, \mathrm{h}_{3}=0 \\
\ln \left(\mu_{3} / \mu_{1}\right)=-1.646 \\
\gamma \mathrm{a}=-1.646\end{array}$ & 1.9471 & 1.9178 & 0.4102 & 0.2888 & 3.9598 & 3.7613 \\
\hline $\begin{array}{l}\mathrm{h}_{1}=\mathrm{a}, \mathrm{h}_{2}=0.771 \mathrm{a}, \\
\mathrm{h}_{3}=0.229 \mathrm{a} \\
\begin{array}{l}\ln \left(\mu_{3} / \mu_{1}\right)=-1.646 \\
\gamma \mathrm{a}=-2.135\end{array}\end{array}$ & 2.0905 & 2.0336 & .4827 & .3190 & 4.6033 & 4.2371 \\
\hline $\begin{array}{l}\mathrm{h}_{1}=\mathrm{a}, \mathrm{h}_{2}=0.545 \mathrm{a}, \\
\mathrm{h}_{3}=0.455 \mathrm{a} \\
\ln \left(\mu_{3} / \mu_{1}\right)=-1.646 \\
\gamma \mathrm{a}=-3.021\end{array}$ & 2.243 & 2.1690 & .5565 & .3448 & 5.3389 & 4.8236 \\
\hline
\end{tabular}

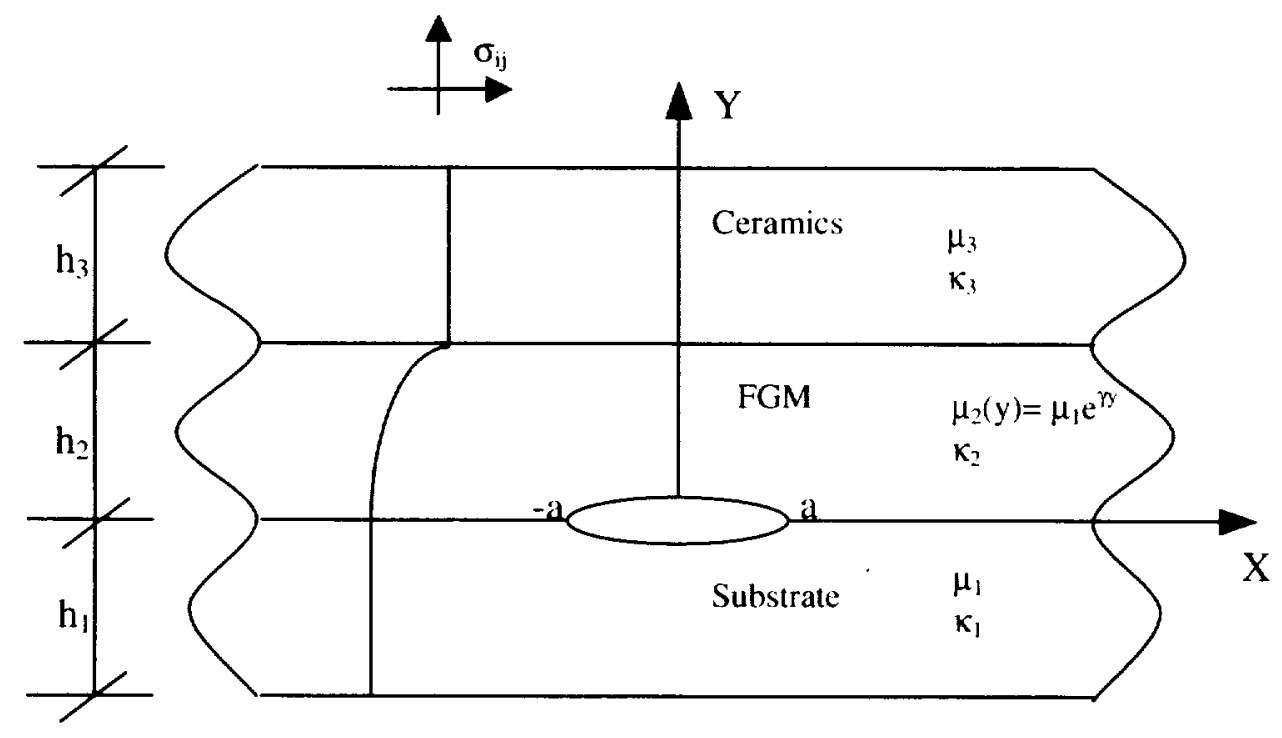

Figure 1. Geometry of the interface crack for a functionally graded layer sandwiched between the homogeneous substrate and coating materials. 


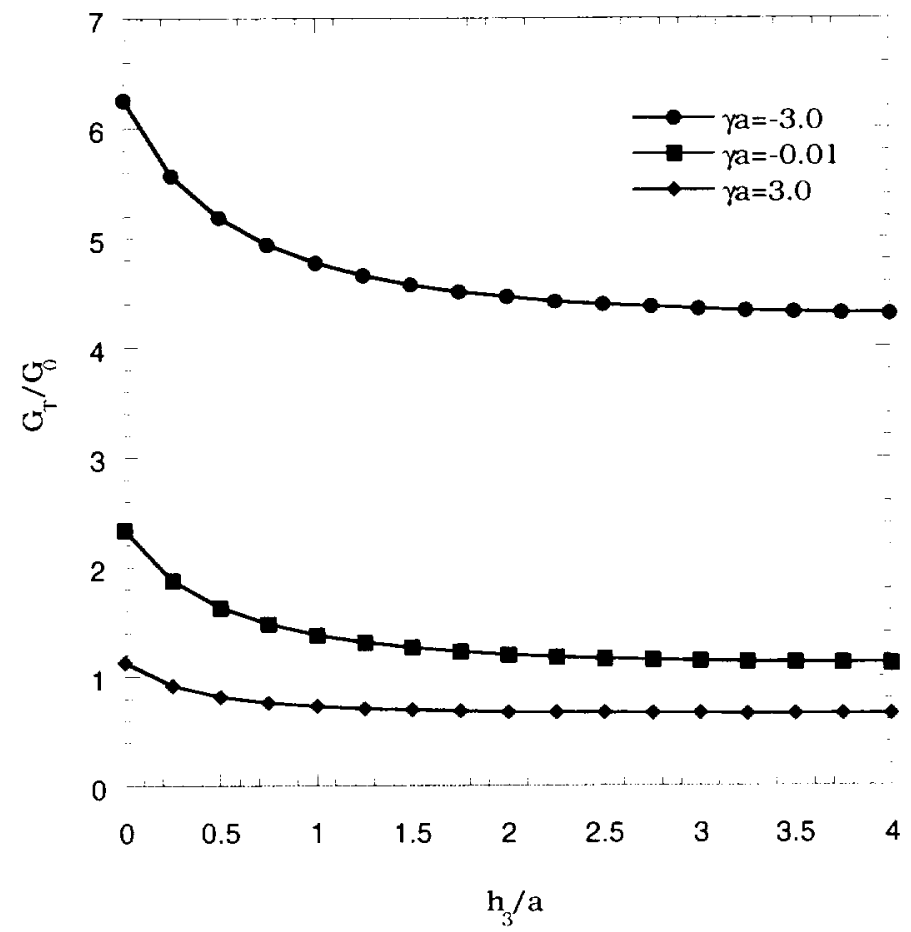

Figure 2. Influence of $h_{3} / a$ on the total SERR for $h_{1} / a=4.0$ and $h 2 / a=1.0$ under loading of uniform normal stress.

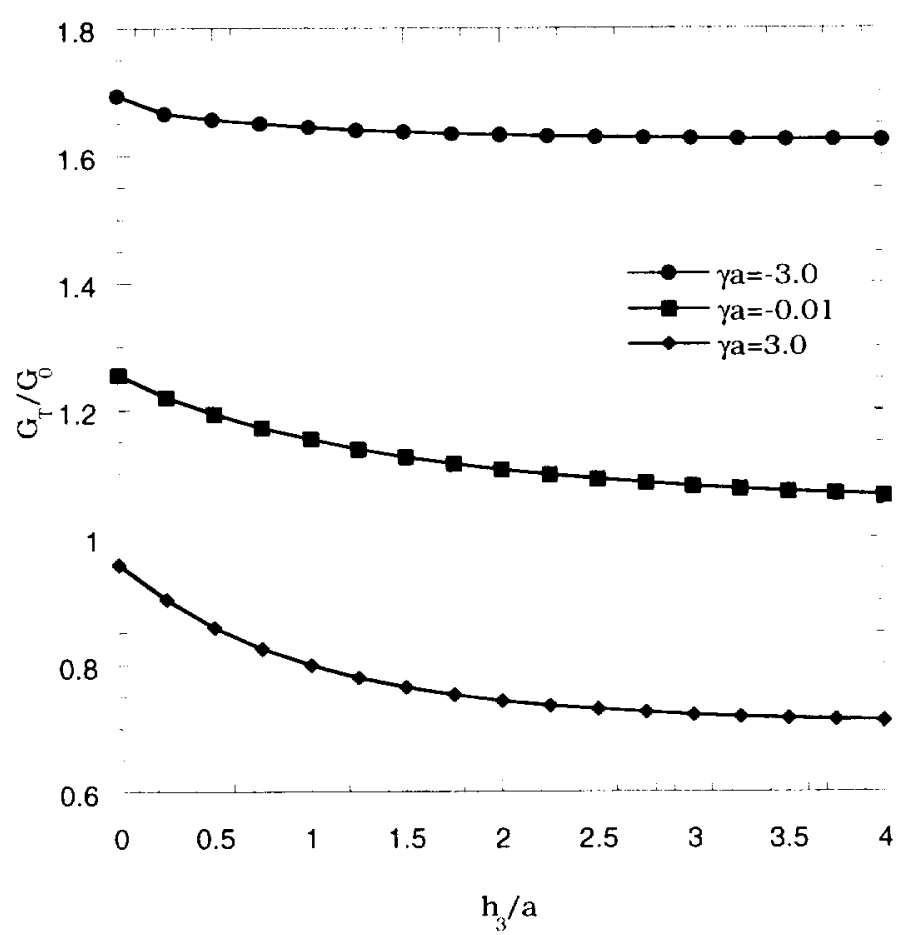

Figure 3. Influence of $h_{3} / a$ on the total SERR for $h_{1} / a=4.0$ and $h 2 / a=1.0$ under loading of uniform shear stress. 


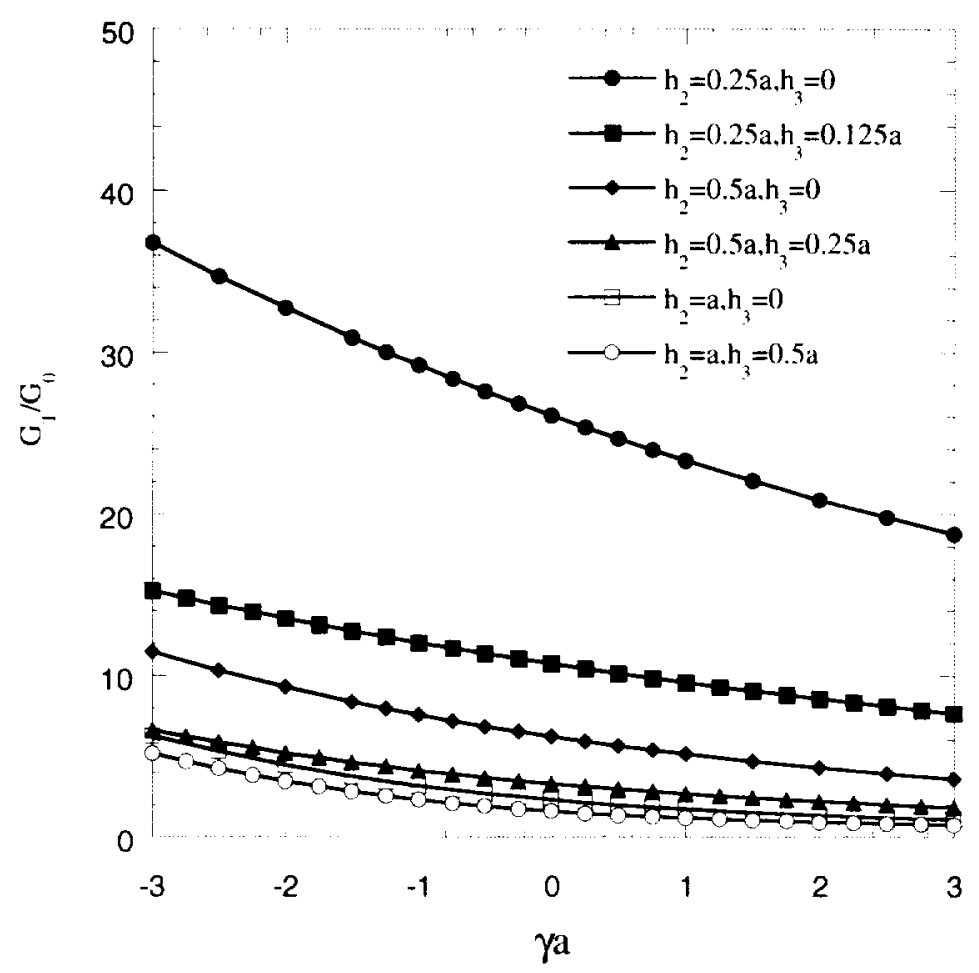

Figure 4. Influence of $h_{2} / a$ and $h_{3} / a$ on the total normalized SERR for $h_{1} / a=100.0$ under loading of uniform normal stress.

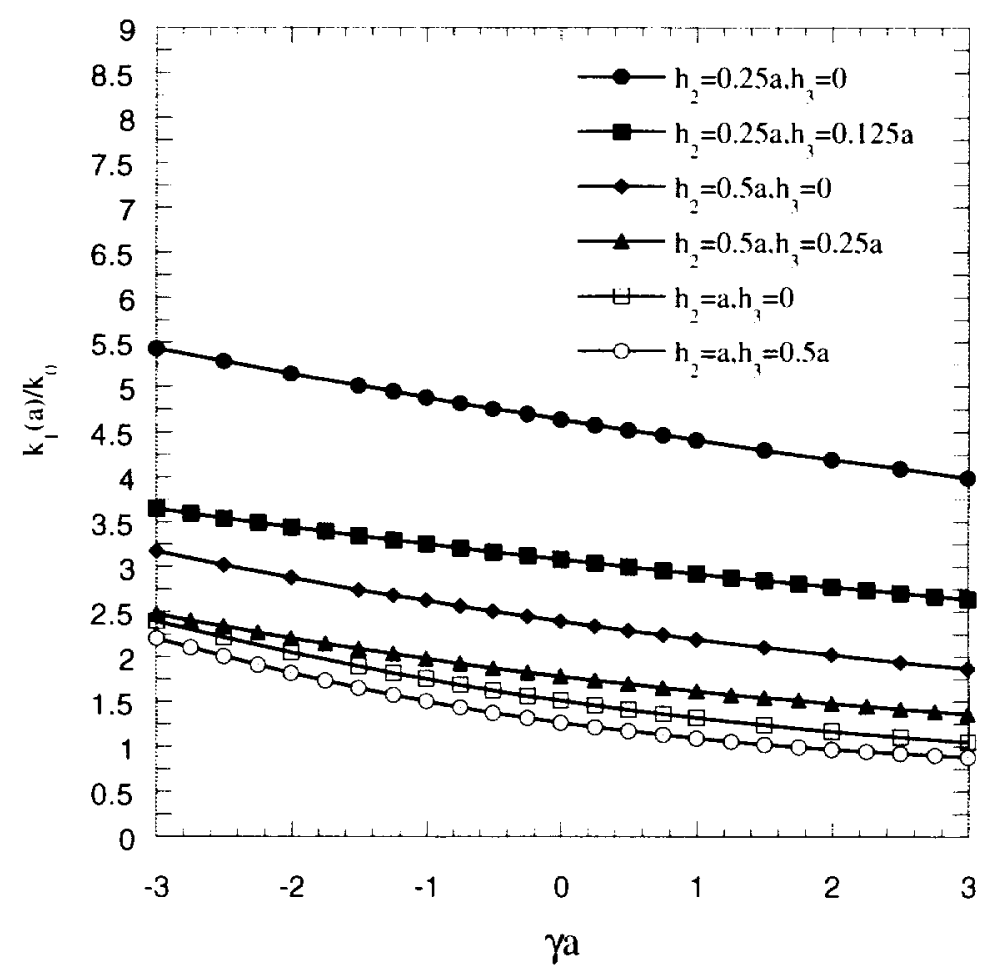

Figure 5. Influence of $h_{2} / a$ and $h_{3} / a$ on the normalized mode I SIF for $h_{1} / a=100.0$ under loading of uniform normal stress. 


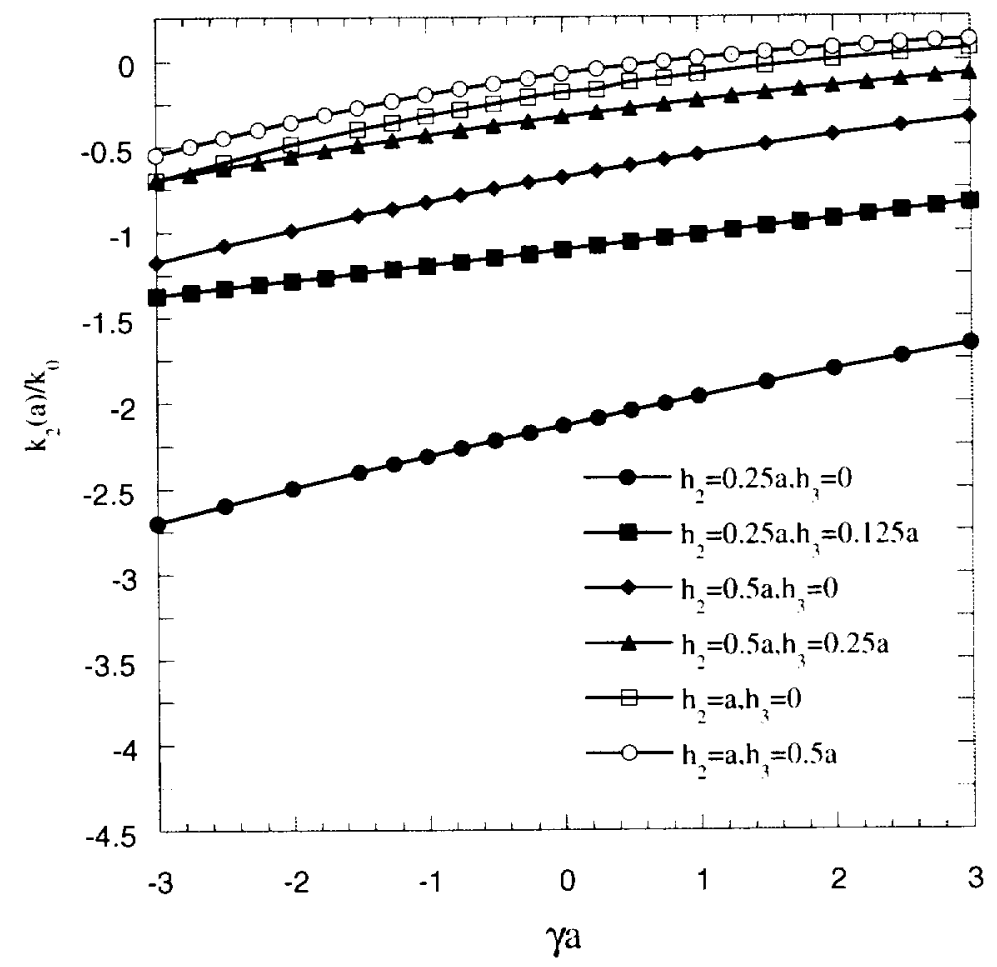

Figure 6. Influence of $h_{2} / a$ and $h_{3} / a$ on the normalized mode II SIF for $h_{1} / a=100.0$ under loading of uniform normal stress.

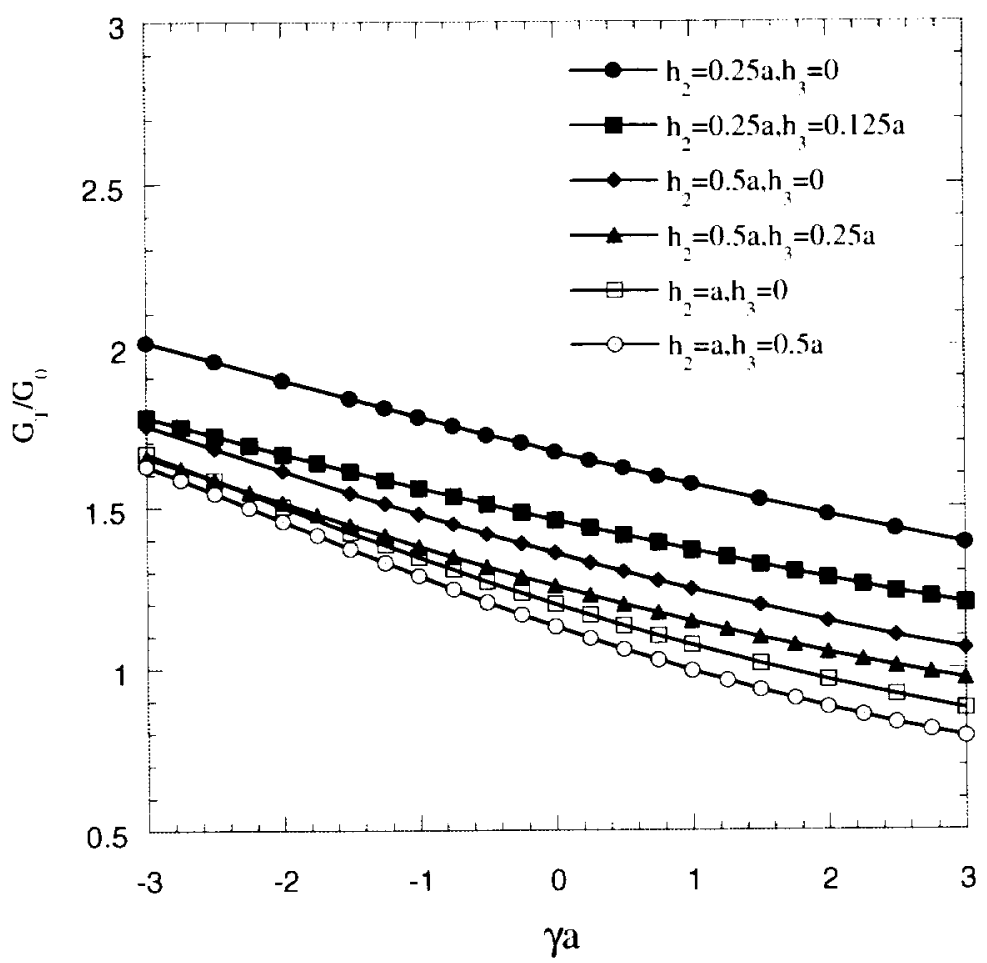

Figure 7. Influence of $h_{2} / a$ and $h_{3} / a$ on the total normalized SERR for $h_{1} / a=100.0$ under loading of uniform shear stress. 


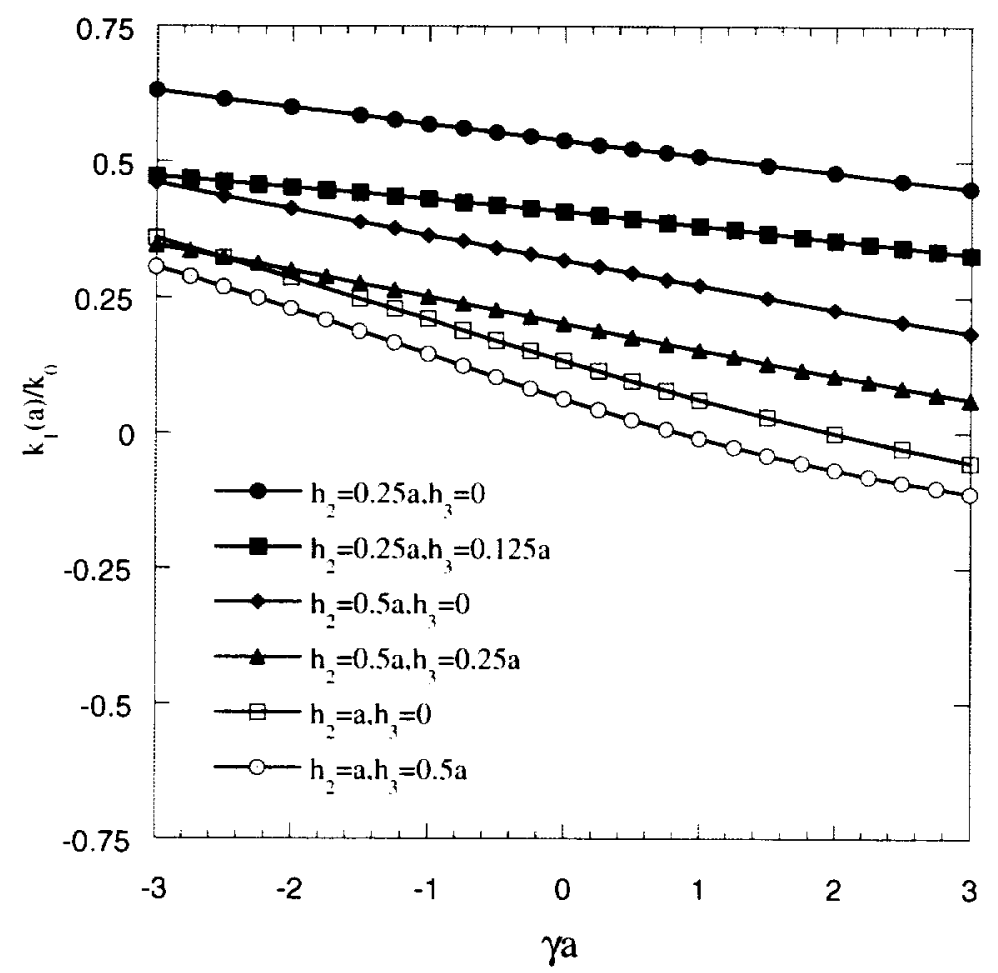

Figure 8. Influence of $h_{2} / a$ and $h_{3} / a$ on the normalized mode I SIF for $h_{1} / a=100.0$ under loading of uniform shear stress.

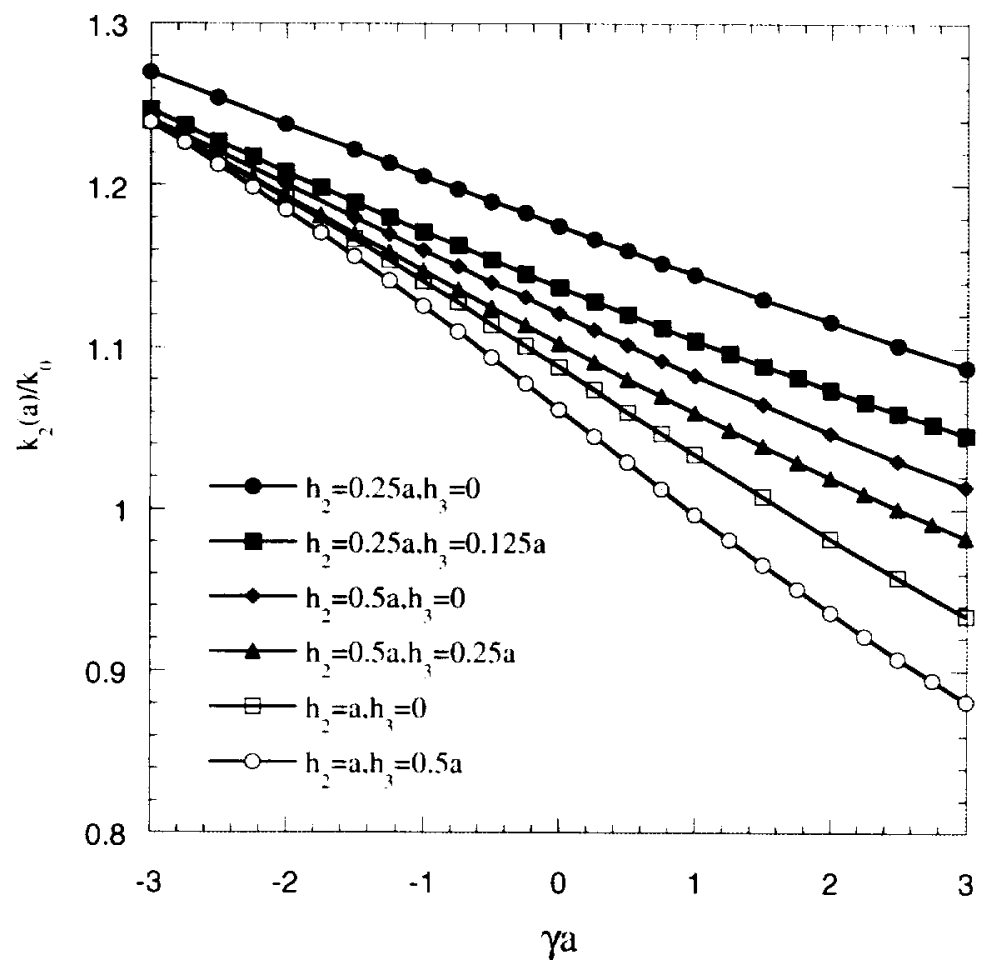

Figure 9. Influence of $h_{2} / a$ and $h_{3} / a$ on the normalized mode II SIF for $h_{1} / a=100.0$ under loading of uniform shear stress. 


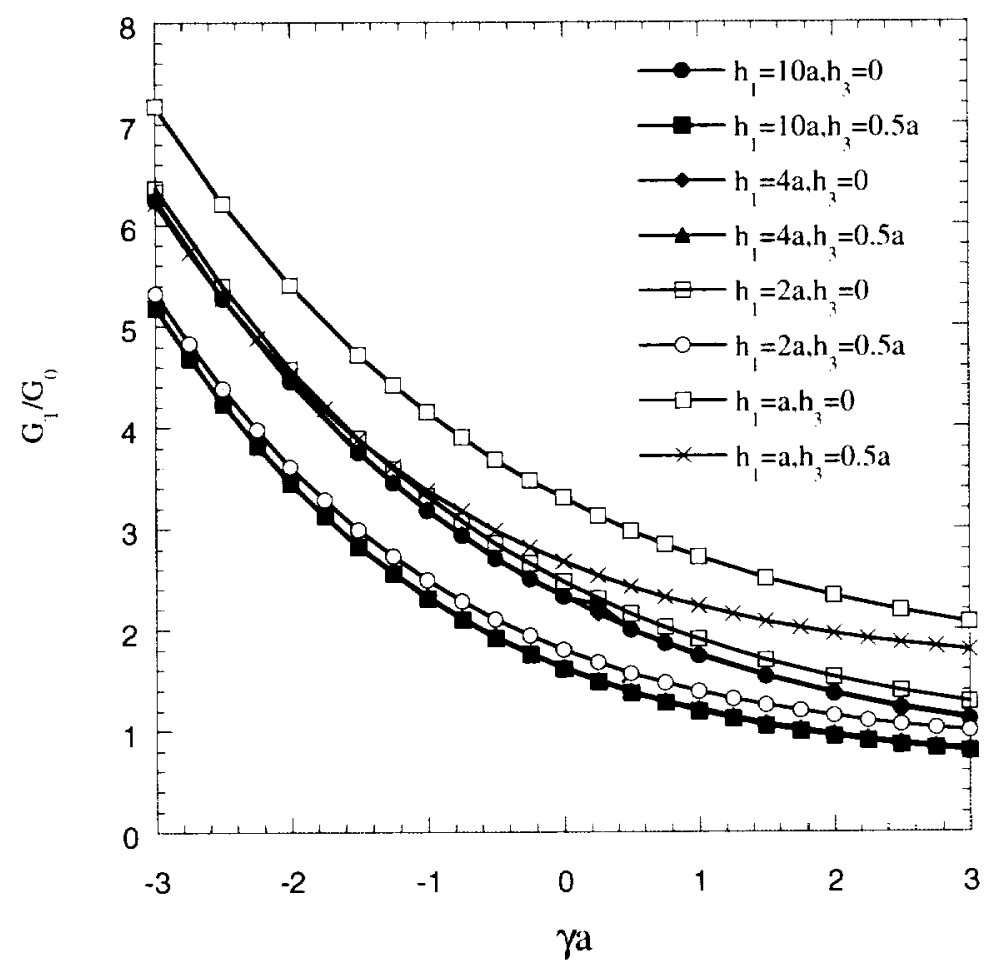

Figure 10. Influence of $h_{1} / a$ and $h_{3} / a$ on the total normalized SERR for $h_{2} / a=1.0$ under loading of uniform normal stress.

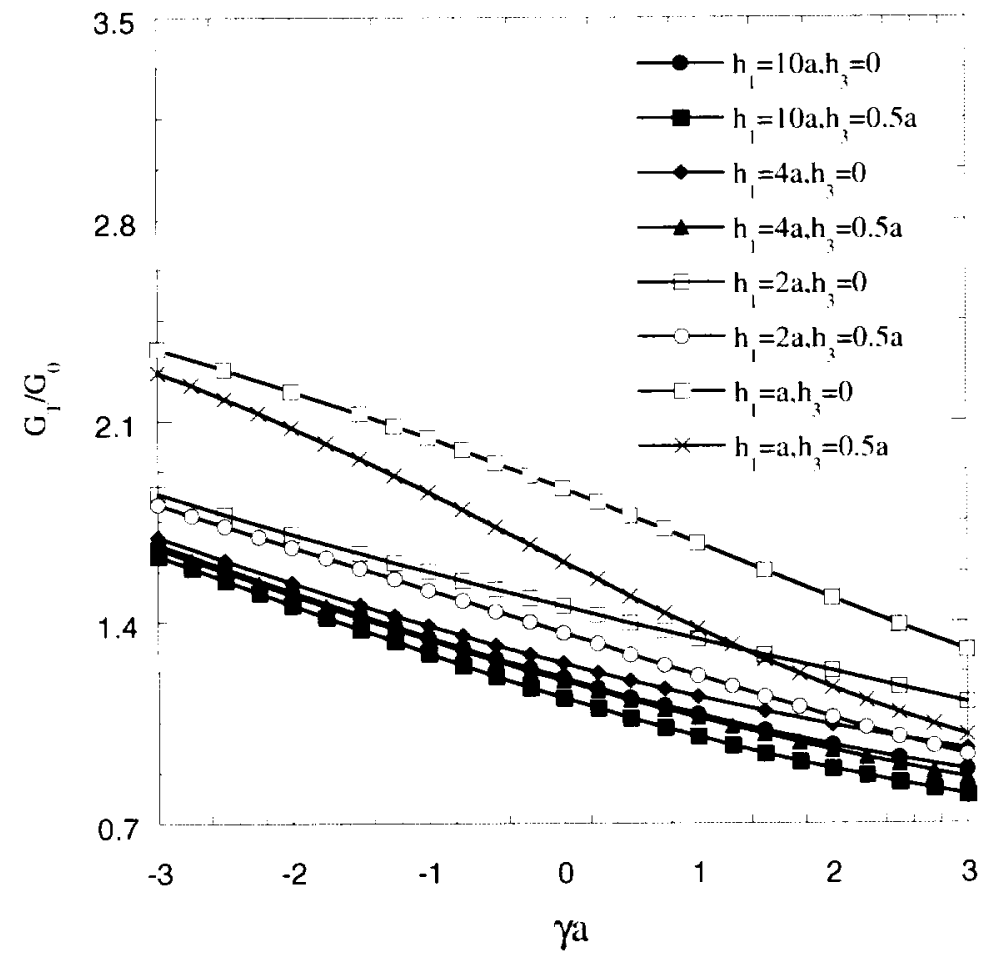

Figure 11. Influence of $h_{1} / a$ and $h_{3} / a$ on the total normalized SERR for $h_{2} / a=1.0$ under loading of uniform shear stress. 


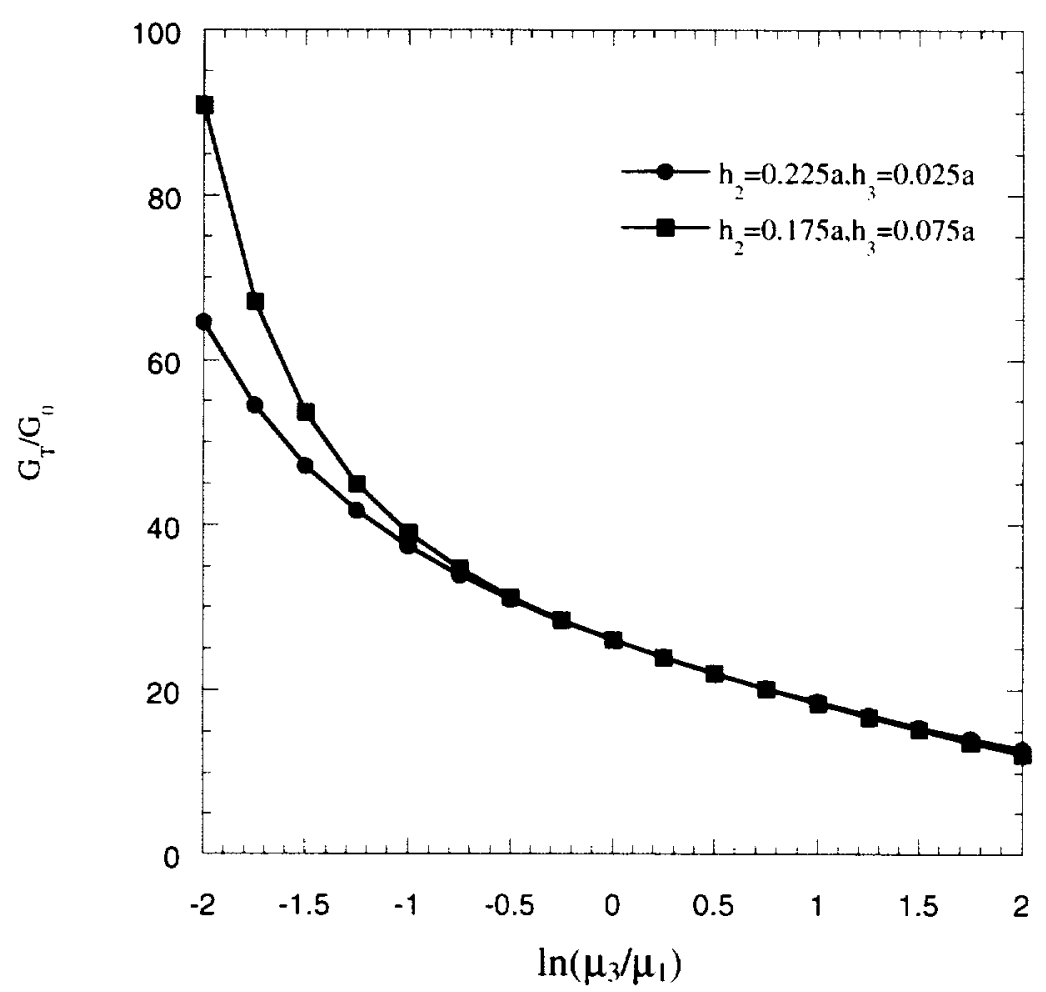

Figure 12. SERR versus $\ln \left(\mu_{3} / \mu_{1}\right)$ for $h_{2}+h_{3}=0.25 \mathrm{a}$ under loading of uniform normal stress. 


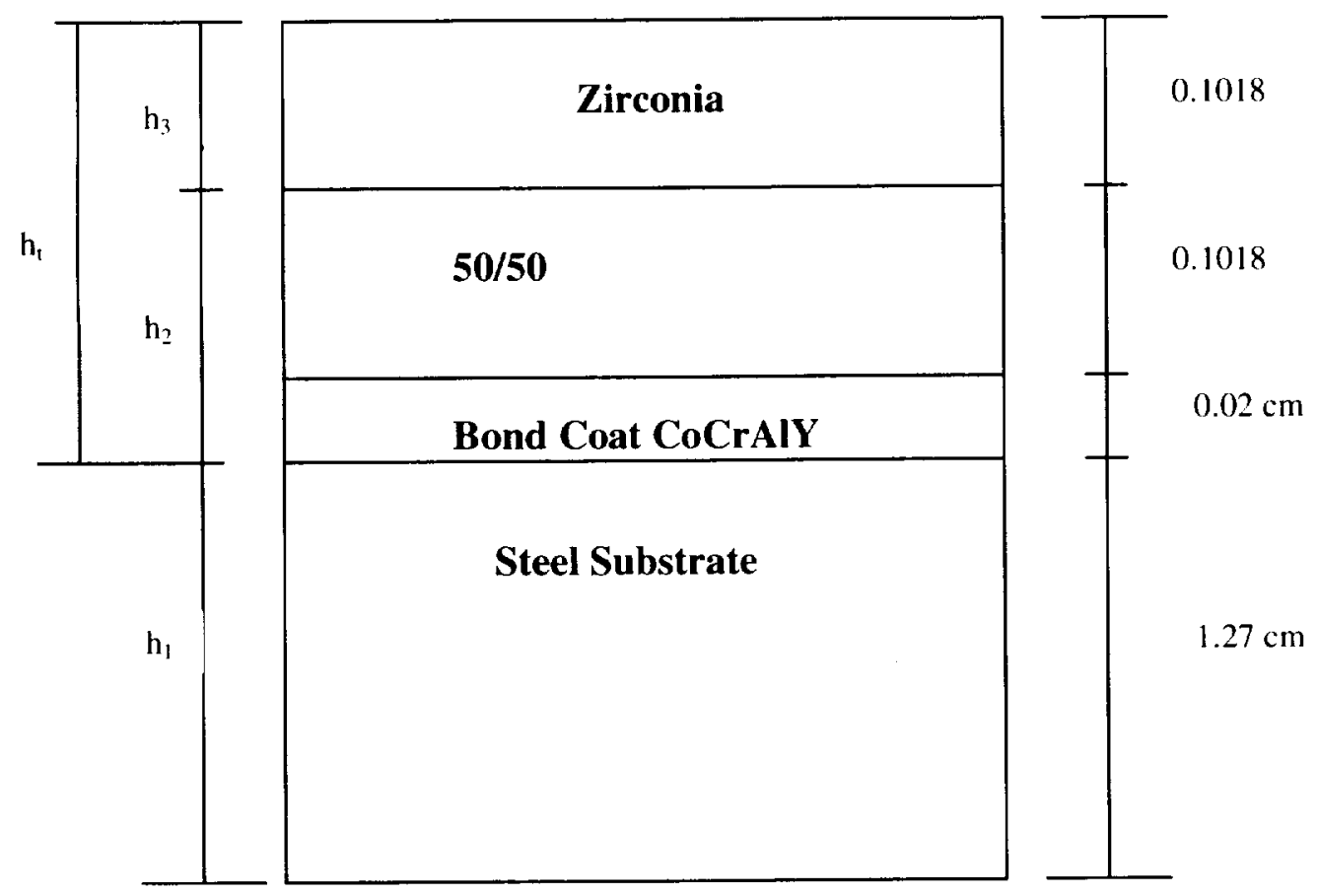

(a)

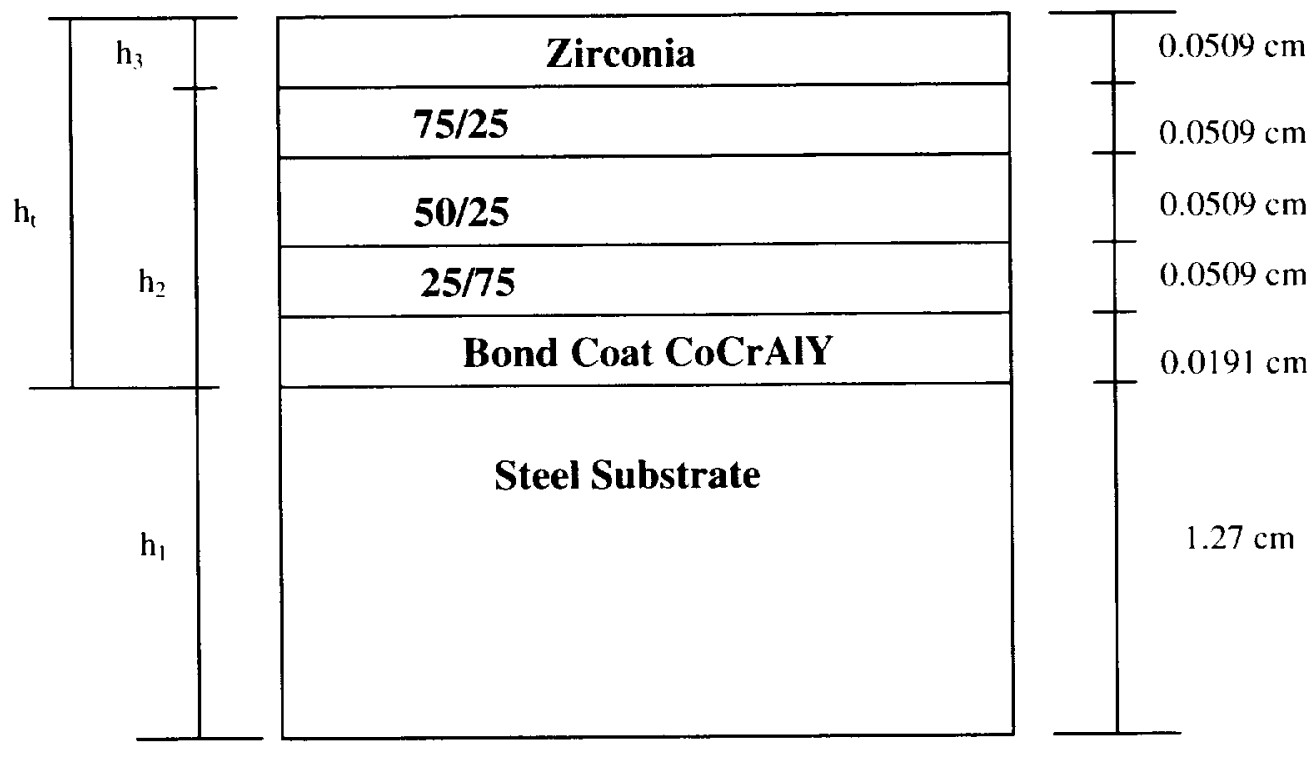

(b)

Figure 13. Geometry of the Zirconia thermal barrier on steel substrate (from Kokini and Choules (1995)), (a) two layer FGM, (b) four layer FGM. 


\section{APPENDIX}

$$
\begin{aligned}
& \text { C11 = }\left(\alpha \left(\left(1-\mathrm{E}^{\mathrm{bla}}+4 \mathrm{E}^{2 \mathrm{hla}} \mathrm{hl} \alpha\right) \mathrm{m}\left(1+x_{1}\right)+\right.\right. \\
& \left.a\left(1+\mathrm{E}^{\mathrm{hla}}-2 \mathrm{E}^{2 \mathrm{hla}}\left(1+4 \mathrm{~h}^{2} a^{2}\right)-\left(-1+\mathrm{E}^{2 \mathrm{hla}}\right)^{2} \mathrm{k}_{1}\right)\right) / / \\
& \left(-4\left(-1+\mathrm{E}^{2 \mathrm{hla}}\right)^{2}+16 \mathrm{E}^{2 \mathrm{hl} \alpha} \mathrm{bl}^{2} \alpha^{2}\right)+ \\
& \frac{1}{8}\left(-a^{2}\left(-3+x_{2}\right)+m_{1}^{2}\left(1+x_{2}\right)\right) ; \\
& C 12=\left(\alpha \left(\left(1-E^{b 2 a}+4 E^{2 h 1 a} h 1 \alpha\right) m_{2}\left(1+x_{1}\right)+\right.\right. \\
& \left.\left.a\left(1+E^{b l a}-2 E^{2 h l a}\left(1+b d^{2} a^{2}\right)-\left(-1+E^{2 h a}\right)^{2} k_{1}\right)\right)\right) /
\end{aligned}
$$

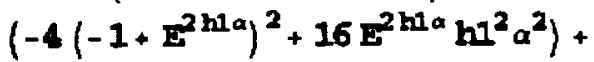

$$
\begin{aligned}
& \frac{1}{8}\left(-\alpha^{2}\left(-3+x_{2}\right)+m_{2}^{2}\left(1+x_{2}\right)\right) \\
& 913=\left(\alpha \left(\left(1-E^{h l a}+4 E^{2 h a} h 1 a\right) m_{\theta}\left(1+x_{1}\right)+\right.\right.
\end{aligned}
$$

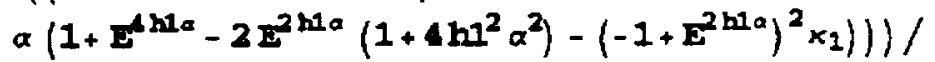

$$
\begin{aligned}
& \left(-4\left(-1+\mathrm{E}^{2 \mathrm{bla}}\right)^{2}+16 \mathrm{z}^{2 \mathrm{hla}} \mathrm{hi}^{2} \mathrm{a}^{2}\right)+ \\
& \frac{1}{8}\left(-\alpha^{2}\left(-3+x_{2}\right)+m^{2}\left(1+x_{2}\right)\right) ; \\
& C 14=\left(a \left(\left(1-z^{h l a}+4 z^{2 h 1 a} h l \alpha\right)\left(1+x_{1}\right)+\right.\right. \\
& \left.\left.\alpha\left(1+E^{h l a}-2 E^{2 h 1 a}\left(1+4 h 1^{2} a^{2}\right)-\left(-1+E^{2 h l a}\right)^{2} x_{1}\right)\right)\right) / \\
& \left(-4\left(-1+\mathrm{E}^{2 \mathrm{hl} \alpha}\right)^{2}+16 \mathrm{E}^{2 \mathrm{hl} \alpha} \mathrm{hI}^{2} \alpha^{2}\right)+ \\
& \frac{1}{8}\left(-a^{2}\left(-3+k_{2}\right)+m^{2}\left(1+x_{2}\right)\right) \\
& C 1=-\left(I \alpha \mid-\alpha\left(-1+L^{h l \alpha}+4 E^{2 h l a} h l \alpha\right)\left(1+x_{1}\right)+\right. \\
& \left.m\left(1+E^{\text {hla }}-2 \mathrm{E}^{2 \mathrm{hla}}\left(1+4 \mathrm{hI}^{2} \alpha^{2}\right)-\left(-1+\mathrm{E}^{2 \mathrm{hl} a}\right)^{2} \kappa_{1}\right)\right) / \\
& \left(-4\left(-1+\mathrm{E}^{2 \mathrm{hla}}\right)^{2}+16 \mathrm{E}^{2 \mathrm{hla}} \mathrm{hl}^{2} \mathrm{a}^{2}\right)+ \\
& \frac{I a\left(m_{1}^{2}\left(-3+k_{2}\right)-a^{2}\left(1+k_{2}\right)\right)}{B\left(-\gamma+m_{1}\right)} \\
& 022=-\left\{I \alpha \left(-\alpha\left(-1+\mathrm{E}^{h \mathrm{hl}^{\alpha}}+4 \mathrm{I}^{2 \mathrm{hl \alpha}} \mathrm{hl} \alpha\right)\left(1+x_{1}\right)+\right.\right. \\
& \left.\operatorname{mg}\left(1+\mathrm{E}^{\mathrm{hl} \alpha}-2 \mathrm{E}^{2 \mathrm{hl} \alpha}\left(1+4 \mathrm{hl}^{2} \alpha^{2}\right)-\left(-1+\mathrm{E}^{2 \mathrm{hl} \alpha}\right)^{2} \times 1\right)\right) / / \\
& \left(-4\left(-1+\mathrm{E}^{2 \mathrm{hla}}\right)^{2}+16 \mathrm{I}^{2 \mathrm{hla}} \mathrm{hl}^{2} a^{2}\right)+ \\
& \frac{I a\left(m_{2}^{2}\left(-3+x_{2}\right)-a^{2}\left(1+x_{2}\right)\right)}{8\left(-\gamma+m_{2}\right)} \\
& C 23=-\left(I a \left(-a\left(-1+E^{A h l a}+4 E^{2 b 1 \alpha} b 1 \alpha\right)\left\langle 1+\kappa_{1}\right)+\right.\right. \\
& \left.\left.m\left(1+\mathrm{E}^{\mathrm{hl} a}-2 \mathrm{E}^{2 \mathrm{hla}}\left(1+4 \mathrm{hl}^{2} \alpha^{2}\right)-\left(-1+\mathrm{E}^{2 \mathrm{hla}}\right)^{2} \times_{1}\right)\right)\right) / \\
& \left(-4\left(-1+\mathrm{E}^{2 \mathrm{hla}}\right)^{2}+16 \mathrm{E}^{2 \mathrm{hla}} \mathrm{hI}^{2} a^{2}\right)+ \\
& \frac{I a\left(m^{2}\left(-3+x_{2}\right)-\alpha^{2}\left(1+x_{2}\right)\right)}{8\left(-\gamma+m_{B}\right)} ;
\end{aligned}
$$

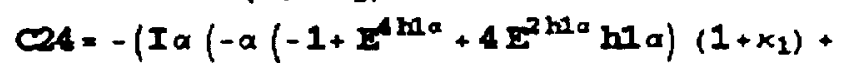

$$
\begin{aligned}
& \left.\left.\operatorname{ma}\left(1+\mathrm{z}^{\mathrm{hla} a}-2 \mathrm{E}^{2 \mathrm{hla}}\left(1+4 \mathrm{hl}^{2} \alpha^{2}\right)-\left(-1+\mathrm{E}^{2 \mathrm{hla}}\right)^{2} \times_{1}\right)\right)\right) / \\
& \left(-4\left(-1+\mathrm{E}^{2 \mathrm{hla}}\right)^{2}+16 \mathrm{H}^{2 \mathrm{hla}} \mathrm{hr}^{2} a^{2}\right) \text {. } \\
& \frac{I \alpha\left(m^{2}\left(-3+x_{2}\right)-\alpha^{2}\left(1+x_{2}\right)\right)}{8\left(-\gamma+x_{4}\right)} \text {; }
\end{aligned}
$$




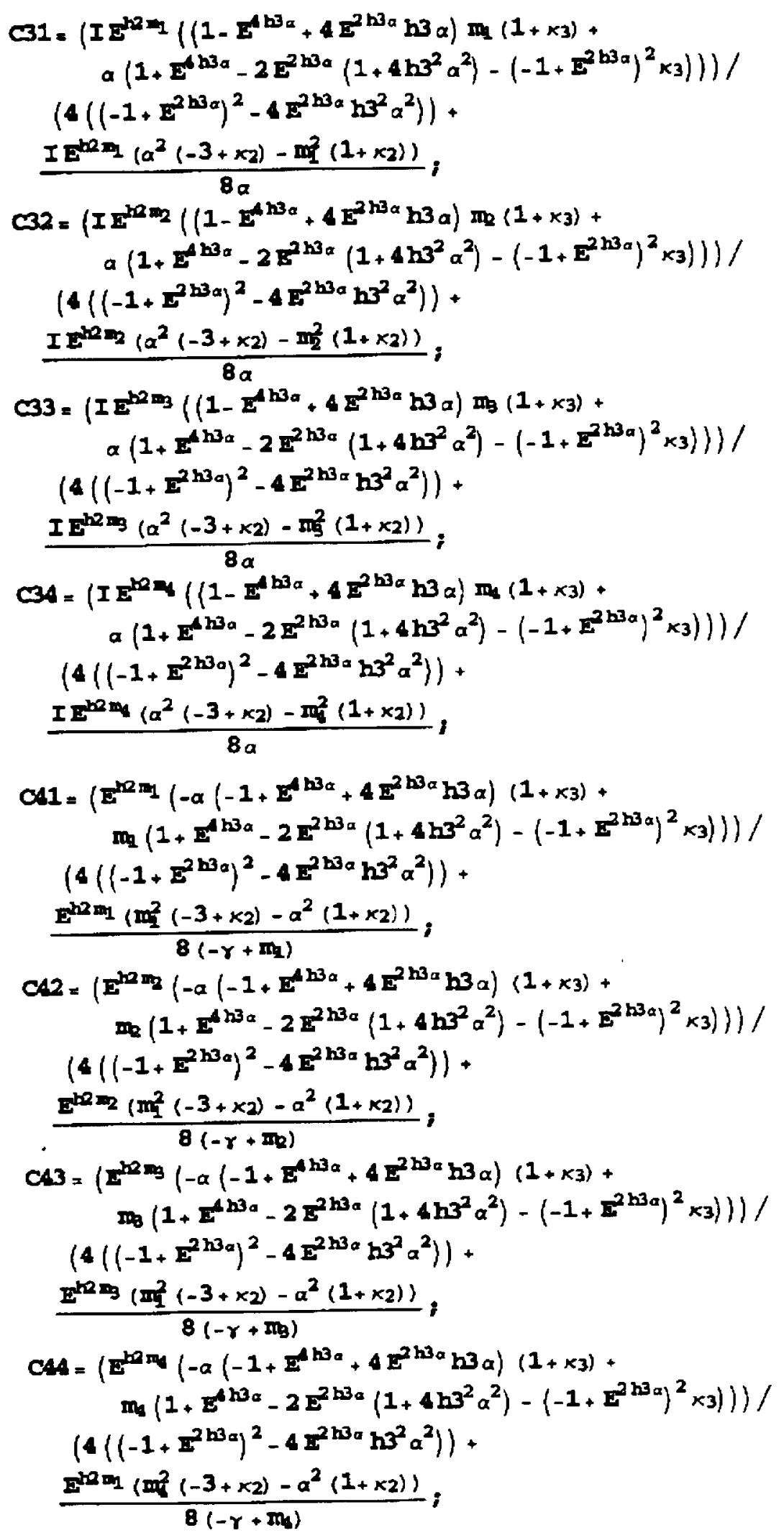




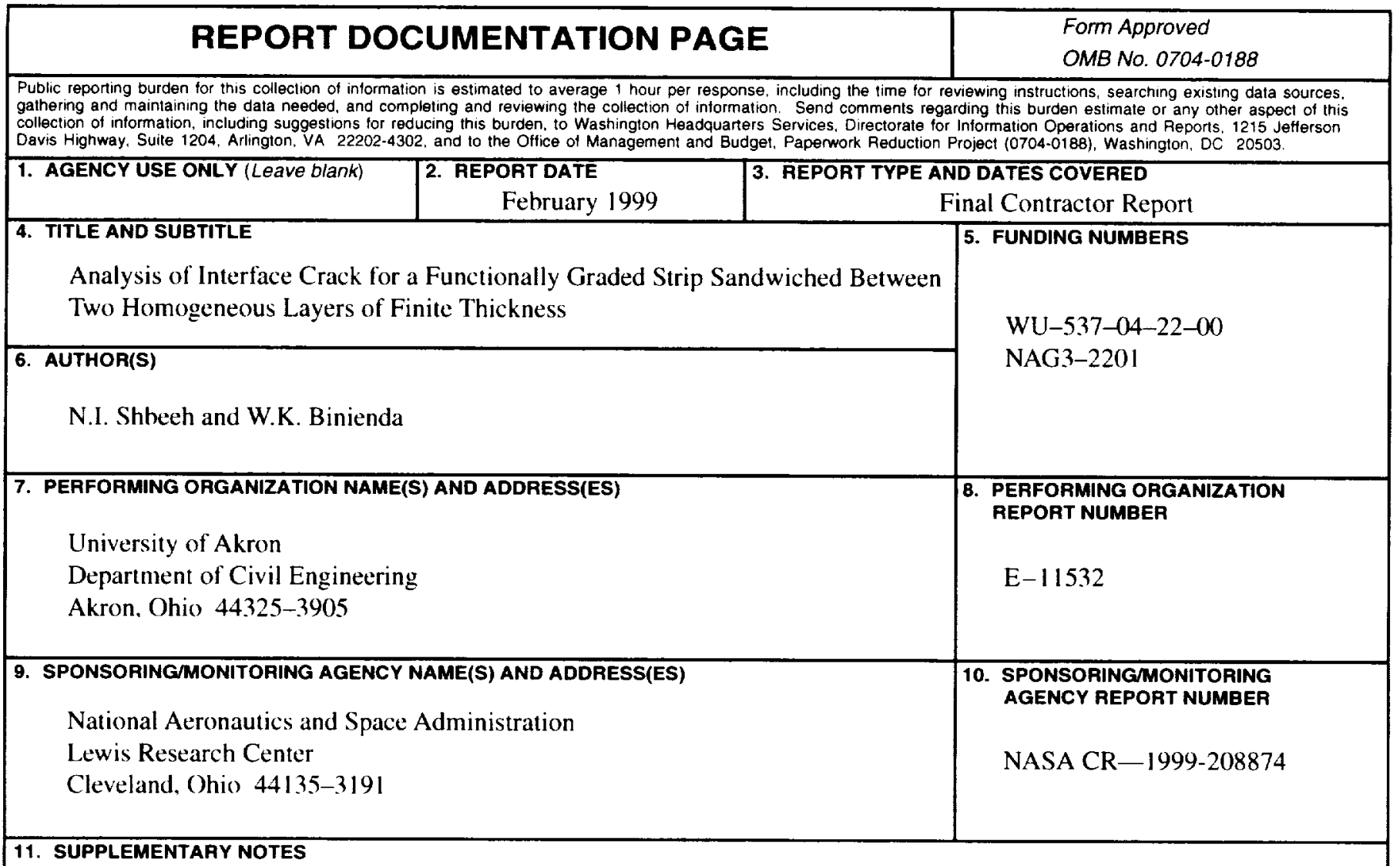

11. SUPPLEMENTARY NC

Project Manager, Gary R. Halford, NASA Lewis Research Center, organization code 5000, (216) 433-3265.

12a. DISTRIBUTIONAVAILABILITY STATEMENT

Unclassified - Unlimited

Subject Category: 39
Distribution: Nonstandard

This publication is available from the NASA Center for AeroSpace Information, (301) 621-0390. 12b. DISTRIBUTION CODE

\section{ABSTRACT (Maximum 200 words)}

The interface crack problem for a composite layer that consists of a homogeneous substrate, coating and a nonhomogeneous interface was formulated for singular integral equations with Cauchy kernels and integrated using the Lobatto-Chebyshev collocation technique. Mixed-mode Stress Intensity Factors and Strain Energy Release Rates were calculated. The Stress Intensity Factors were compared for accuracy with relevant results previously published. The parametric studies were conducted for the various thickness of each layer and for various non-homogeneity ratios. Particular application to the Zirconia thermal barrier on steel substrate is demonstrated.

14. SUBJECT TERMS

Thermal barrier coating; Functionally graded material; Crack propagation; Strain energy release rate; Stress intensity factors; Singular integral equations; Collocation method

\begin{tabular}{|c|c|c|}
\hline $\begin{array}{c}\text { 17. SECURITY CLASSIFICATION } \\
\text { OF REPORT } \\
\text { Unclassified }\end{array}$ & $\begin{array}{c}\text { 18. SECUAITY CLASSIFICATION } \\
\text { OF THIS PAGE } \\
\text { Unclassified }\end{array}$ & $\begin{array}{c}\text { 19. SECURITY CLASSIFICATION } \\
\text { OF ABSTRACT } \\
\text { Unclassified }\end{array}$ \\
\hline
\end{tabular}

